\title{
Raffinage des huiles et des corps gras et élimination des contaminants
}

\author{
Xavier PAGES \\ Odile MORIN \\ Céline BIROT \\ Marie GAUD \\ Stéphane FAZEUILH \\ Morgan GOUBAND \\ ITERG,
institut des corps gras,
11, rue Gaspard-Monge,
33600 Pessac,
France (www.iterg.com)
$<x$.pages@iterg.com>
$<$ o.morin@iterg.com>
}

\section{Raffinage des corps gras : objectifs, procédés}

Le raffinage a pour but de maintenir ou d'améliorer les caractères organoleptiques (goût et odeur neutres, limpidité, couleur jaune clair), nutritionnels et la stabilité des corps gras. Pour ce faire, il met en œuvre plusieurs étapes pour éliminer des composés indésirables (gommes, cires, acides gras libres, pigments, traces métalliques, composés odorants volatils) et les contaminants potentiellement présents dans les matières premières, tout en maîtrisant la formation de nouveaux composés indésirables par hydrolyse, oxydation ou isomérisation. La conduite du procédé peut au besoin s'adapter aux usages ultérieurs qui seront faits des huiles raffinées produites: un usage alimentaire (ou cosmétique/pharmaceutique) conduira le raffineur à optimiser son procédé afin de conserver les constituants d'intérêt nutritionnel (acides gras polyinsaturés, vitamine E) ; pour un usage technique, le procédé s'adaptera aux emplois visés (par exemple, dans le cas d'un procédé ultérieur de transestérification pour la production d'esters méthyliques d'huiles végétales, biocarburant).

II existe deux types de raffinage: chimique et "physique», qu'il conviendrait mieux

\begin{abstract}
Refining is a powerful technology to guarantee a high food grade quality of the refined oils and fats. This overview of many of the safety challenges related to different contaminant residues, the refining processes have to and actually do respond to, focuses for each contaminant (pesticides, polycyclic hydrocarbons, other compounds as extraction solvent, metallic element traces, aflatoxins, dioxins and polychlorobiphenyls, and new appearing contaminants like phthalates and 3-monochloropropanediol esters) on their nature, origin, occurrence and regulatory status before describing the required conditions of their elimination by chemical and "physical" refining with the mention of the most efficient refining step as known from the GMP or identified from original experimental works. Identifying the physicochemical characteristics (hydro-solubility, lipophilic property, molecular weight and vapour pressure) indicative of a potential contaminant behaviour during refining is an interesting preliminary orientation tool. The quality target for refined oils and fats include on one hand the control and optimisation of organoleptic and nutritional characteristics as well as stability during further storage, and on the other hand, the elimination of contaminants avoiding or controlling the formation of new undesirable compounds. For each contaminant to be eliminated, the technological issues have to be found at the crossroads of these demands.
\end{abstract}

Key words: vegetable oils and fats, chemical refining, physical refining, contaminant, food safety, regulation

d'appeler « distillation neutralisante ». Le raffinage chimique élimine les acides gras libres par une étape de neutralisation à la soude ; la distillation neutralisante élimine ces composés indésirables (désacidification) par distillation sous vide poussé avec injection de vapeur.

Les figures 1 et 2 présentent respectivement les différentes étapes d'un raffinage par voie chimique et par distillation neutralisante, en précisant les principaux composés éliminés à chaque étape.

À côté de ces principaux composés, chaque étape contribue à l'élimination d'autres substances indésirables, de contaminants potentiels et des coproduits de l'étape précédente. Les préoccupations récurrentes en matière de sécurité sanitaire des produits raffinés réclament de vérifier systématiquement les conditions d'élimination des différents contaminants susceptibles de polluer les huiles brutes, et cela, régulièrement afin d'intégrer les nouvelles substances identifiées comme polluants potentiels. L'ITERG, depuis plus de dix ans, a donc inscrit à son programme de recherche l'étude des conditions d'élimination des contaminants par raffinage chimique ou par distillation neutralisante à l'aide de ses installations pilotes ${ }^{1}$; ces études sont logique- ment associées aux travaux de recherche analytique de ses laboratoires d'analyse qui visent la mise au point de méthodes de dosage de ces substances adaptées aux matrices grasses $^{2}$.

\section{Élimination des résidus phytosanitaires}

Les pesticides (aussi appelés produits phytopharmaceutiques ou phytosanitaires) sont des substances chimiques, naturelles ou de synthèse, utilisées en agriculture pour contrôler les différentes sortes de nuisibles (animaux, végétaux, micro-organismes) et ainsi augmenter le rendement des principales cultures. Pour leur application, régie par des conditions strictes intégrées dans les bonnes pratiques culturales, les substances actives sont généralement

\footnotetext{
${ }^{1}$ Tous les essais menés sur ces installations sont conduits le plus souvent en discontinu, ce qui permet de tester les paramètres importants du raffinage (températures, durées, etc.) qui peuvent prendre des valeurs plus extrêmes que dans la réalité industrielle.

${ }^{2}$ Voir contribution de Lacoste et al. dans ce dossier : "Détection de contaminants dans les huiles végétales : bilan à fin $2009 »$.
} 


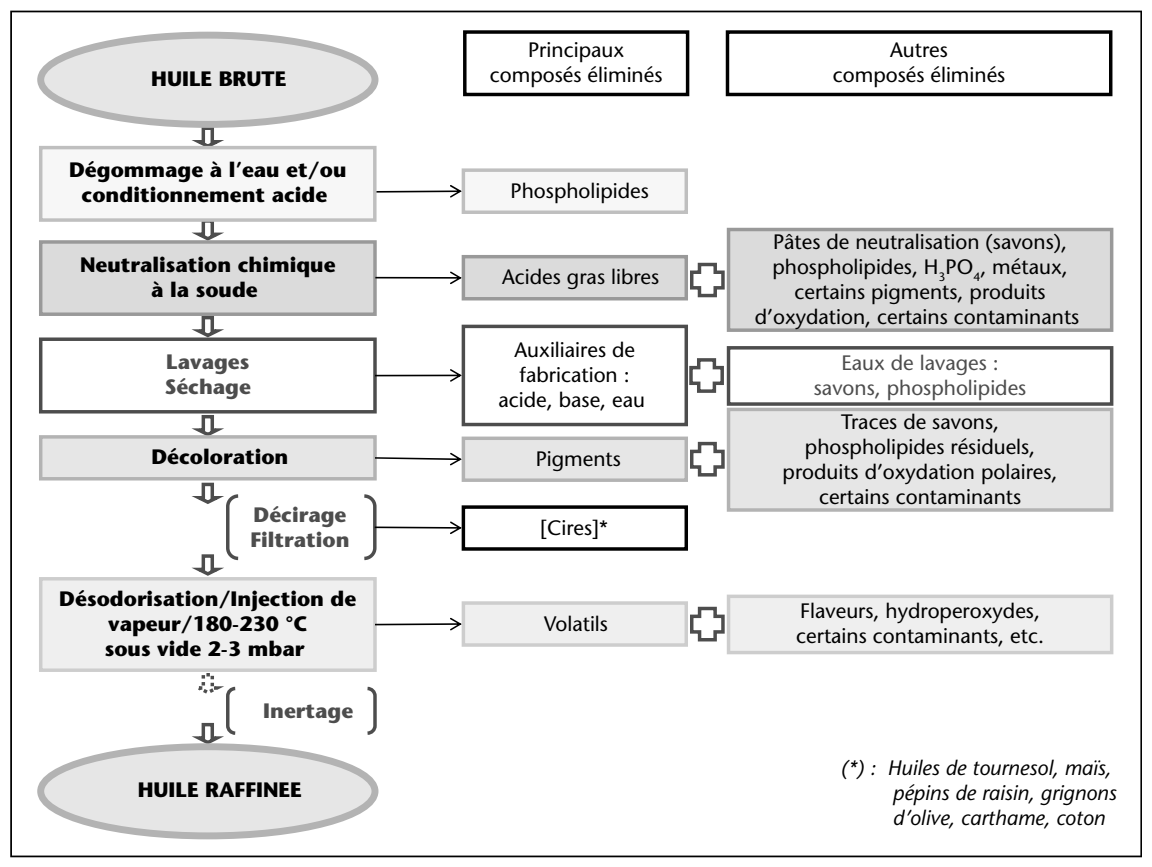

Figure 1. Raffinage par voie chimique : étapes et composés éliminés.

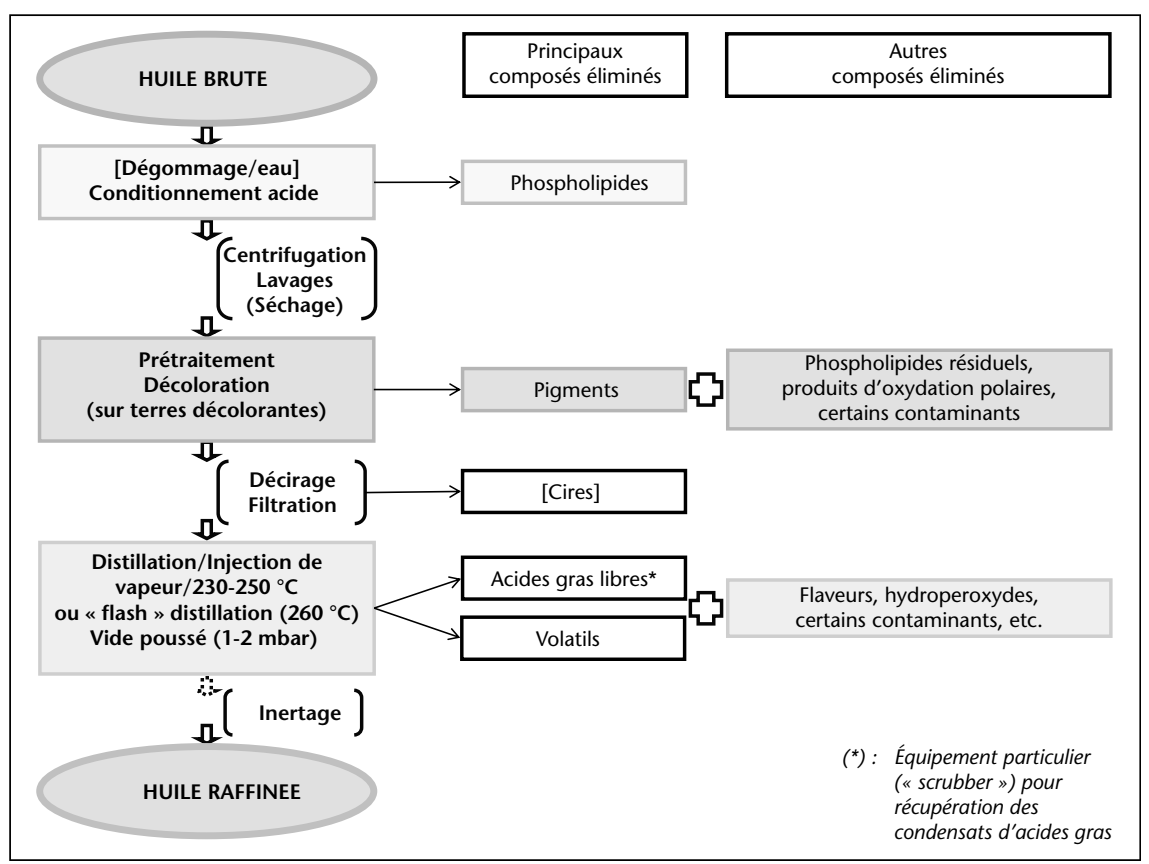

Figure 2. Raffinage par distillation neutralisante (raffinage " physique ») : étapes et composés éliminés.

formulées avec des porteurs ou des correcteurs (solvants, émulsifiants, agents mouillants, adhésifs et agents odoriférants). Les principales familles de substances actives (composés organochlorés, organophosphorés, carbamates et thiocarbamates, pyréthrines et pyréthroïdes, triazines et fumigants) sont utilisées dans les différentes classes de pesticides qui sont employées dans deux grands types de traitements :

- au champ, en prérécolte (herbicides, insecticides, fongicides) : globalement, les composés utilisés actuellement ont une rémanence relativement limitée, par rapport aux pesticides organochlorés utilisés par le passé. En outre, les doses d'application au champ ont considérablement diminué depuis une quinzaine d'années. Les traces retrouvées viennent souvent du non-respect du mode d'emploi des pesticides ;

- en post-récolte (insecticides) : ces traitements concernent notamment l'utilisation d'insecticides (essentiellement à base de composés organophosphorés) sur les grains de céréales en stockage. Les risques de résidus sont ici plus importants, la dégradation des produits appliqués étant nettement plus faible que dans les milieux naturels. Le traitement des locaux de stockage et outils de manutention peuvent également être une source de contamination croisée (céréales/oléagineux).

Les caractéristiques des pesticides sont accessibles dans les ouvrages de référence : The Pesticide Manual et I'Index phytosanitaire (Couteux A, Salaün C, 2009 ; rééditions annuelles).

En matière d'encadrement réglementaire, I'Union européenne a procédé, depuis la parution du règlement (CE) $n^{0} 396 / 2005^{3}$, à une harmonisation des limites maximales en résidus de pesticides (LMR) dans les produits agricoles ${ }^{4}$, l'ensemble étant applicable depuis 2008. Ces LMR s'appuient sur les bonnes pratiques culturales et sont spécifiques à chaque production. Elles sont accessibles dans la base de données de l'UE (DG Sanco) sur les pesticides par produit ou par pesticide ${ }^{5}$; en complément à cette base européenne, FEDIOL donne accès sur son site aux LMR des pesticides dans les oléagineux, les huiles et les corps gras pour I'alimentation humaine et animale à partir des recommandations du Codex Alimentarius où telles qu'elles sont fixées au Brésil et en Argentine $^{6}$.

Le règlement (CE) n 396/2005 modifié, ne fixant les LMR que dans les produits agricoles, prévoit une annexe $\mathrm{VI}$ qui devrait concerner les LMR applicables aux produits transformés et/ou composites et mentionner les facteurs

\footnotetext{
${ }^{3}$ Règlement (CE) n ${ }^{\circ}$ 396/2005 du Parlement et du Conseil du 23 février 2005 concernant les LMR présents dans ou sur les denrées alimentaires et les aliments pour animaux d'origine végétale et animale et modifiant la directive 91/414/CEE du Conseil (JO, 16/3/2005, L70, p. 1-16).

${ }^{4}$ Site EUROPA, synthèse de la législation de I'UE, sécurité alimentaire, contrôles phytosanitaires http://europa.eu/legislation_summaries/food_safety /plant_health_checks/121289_fr.htm et texte consolidé du règlement (CE) $n^{0} 396 / 2005$ (mise à jour 2008 : règlements [CE] $n^{\circ} 839 / 2008$ et 256/2009 non intégrés à ce jour) http://eur-lex.europa.eu/ LexUriServ/LexUriServ.do?uri=CONSLEG:2005R0396: 20080410:FR:PDF

${ }^{5} \mathrm{http}: / /$ ec.europa.eu/sanco_pesticides/public/index.cfm

${ }^{6}$ http://www.fediol.be/8/pesticides.xls
} 
de concentration ou de dilution spécifiques applicables à certaines opérations de transformation, et/ou de mélange ou à certains produits transformés ; la Commission européenne souhaite compléter cette annexe sans avoir encore fixé de calendrier pour cette tâche. En ce qui concerne les huiles végétales, la DG Sanco n'est pas favorable à fixer un facteur de transfert simplement proportionnel à la teneur en huile des oléagineux et réclame des données pour établir des valeurs par défaut. Van Duijn (2008) aborde cette question des facteurs de concentration et présente des résultats de transfert pour l'endosulfan des graines de soja à l'huile brute observés en trituration industrielle; partant du principe qu'un résidu de substance active présent dans un oléagineux se concentrera dans I'huile en fonction de son degré de solubilité dans cette matrice ou dans l'hexane dans le cas d'un procédé d'extraction, il définit le facteur maximum de concentration de la graine à l'huile brute comme $X(\max )=100 / C_{\text {huile }}$ où $C_{\text {huile }}$ représente la teneur en huile de la graine; dans le cas du soja contenant en moyenne $20 \%$ $d^{\prime}$ huile, le facteur $X(\max )$ est donc proche de 5. L'examen de la moyenne des teneurs résiduelles en endosulfan dans 33 lots de graines de soja $(0,068 \mathrm{mg} / \mathrm{kg}$ - rappel, LMR: $0,5 \mathrm{mg} / \mathrm{kg}$ ) et de la moyenne des teneurs résiduelles dans les huiles brutes correspondantes $(0,329 \mathrm{mg} / \mathrm{kg})$ conduit à un facteur de concentration de 4,8 , très proche du facteur théorique $X(\max )$.

\section{Occurrence des résidus de pesticides dans les graines oléagineuses et dans les huiles brutes}

Dauguet et al. (2006) ; Lacoste et al. (2005) ont publié les résultats d'enquêtes menées en France en préalable à la mise en place d'observatoires de la qualité des oléagineux et des huiles brutes (tournesol, colza) en matière de résidus d'insecticides. Le malathion, le pyrimiphos-méthyl et le dichlorvos (insecticides organophosphorés utilisés au stockage) étaient les trois molécules les plus fréquemment détectées dans les graines (20 à $30 \%$ des cas) et dans les huiles brutes (70-80\% des cas), à des teneurs relativement basses pour ces dernières comprises en moyenne entre 0,1 et $0,25 \mathrm{mg} / \mathrm{kg}$ pour l'ensemble de ces trois molécules et des teneurs maximales pouvant atteindre $1 \mathrm{mg} / \mathrm{kg}$ (Lacoste et al., 2005). Les résultats de 2004 montraient que toutes les huiles brutes contenaient au moins un résidu de pesticide de teneur supérieure à la limite de quantification mais que, le plus fréquemment, au moins deux (colza) ou trois (tournesol) résidus étaient présents dans les échantillons collectés.
Lors de la trituration (extraction à I'hexane), ces molécules lipophiles se retrouvent essentiellement dans I'huile brute et très peu dans les tourteaux; donc ce transfert dans l'huile $s^{\prime}$ effectue selon un facteur de concentration de l'ordre de $2^{7}$ (Dauguet et al., 2006).

Les teneurs retrouvées étant relativement basses, I'origine de ces résidus est principalement attribuée à des contaminations croisées du fait que les installations de stockage (communes aux oléagineux et aux céréales) et les matériels de manutention (convoyeurs, élévateurs, etc.) font l'objet de nettoyages et de traitements insecticides, créant ainsi un environnement où ces insecticides sont présents de manière rémanente.

Van Duijn (2008) a publié une compilation de résultats de surveillance obtenus sur trois ans (période 2001-2003) de la qualité des huiles brutes de colza, de tournesol, de soja, de palme, de palmiste et de coco (coprah) entrant en raffinage industriel.

\section{Élimination des résidus de pesticides au raffinage}

Les résultats des enquêtes de surveillance rappelés plus haut démontrent la nécessité des opérations de raffinage pour éliminer les résidus présents dans les huiles brutes.

Pages et al. $(2004,2006)$ ont communiqué les résultats des travaux menés à l'ITERG sur l'efficacité des différentes étapes du raffinage. Ces études, réalisées sur les lignes pilotes des installations du site de Pessac, ont consisté à doper des huiles brutes de colza ou de tournesol (niveaux compris entre 0,2 et $5 \mathrm{mg} / \mathrm{kg}$ ) en différentes substances actives (composés organophosphorés [dichlorvos, malathion, pyrimiphos-méthyl, chlorpyriphos-méthyl, fénitrothion], organochlorés [ $\alpha$ et $\beta$ endosulfan] et pyréthroïde [deltaméthrine]) et à suivre leur devenir au cours de raffinage par voie chimique ou « physique » (distillation neutralisante). Le tableau 1 résume les conditions des différentes étapes des raffinages appliqués aux huiles dopées. Les résultats obtenus sur le comportement des différentes molécules en fonction des traitements mis en œuvre en lien avec certaines de leurs caractéristiques physicochimiques sont présentés ci-après et synthétisés dans le tableau 2.

\section{Conditionnement (acide phosphorique)}

Efficace pour les molécules instables en milieu acide ; parmi les composés étudiés, diminution sensible des teneurs uniquement pour le pyrimiphos-méthyl.

\footnotetext{
${ }^{7}$ Rappel : les teneurs moyennes en huile des graines de tournesol et de colza sont de $50 \%$.
}

\section{Neutralisation alcaline ${ }^{8}$}

Efficacité pour les molécules selon leur degré de solubilité en phase aqueuse (conditionnant leur susceptibilité à réagir) et leur instabilité en milieu basique; parmi les composés étudiés, seuls le dichlorvos et le malathion subissent une forte diminution de concentration à cette étape ; les autres molécules, bien que plus ou moins instables en milieu basique, n'étant pas affectées.

\section{Lavages $^{8}$}

Le critère important de l'efficacité de cette étape est la solubilité dans l'eau des pesticides; là encore, seuls le dichlorvos et le malathion subissent une diminution significative lors de ce traitement. Ces résultats sont en accord avec les caractéristiques physicochimiques de ces molécules: solubilité dans l'eau et coefficient

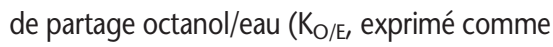
« $\log P »)$, indicateur du caractère plus ou moins lipophile d'une substance ${ }^{9}$ (tableau 2).

\section{Séchage}

Aucun impact significatif n'est observé.

\section{Décoloration}

Les molécules présentant un site cationique apparaissent plus sensibles à l'action des terres décolorantes. Parmi les terres testées, la Tonsil $110 \mathrm{FF}$ donne les meilleurs résultats pour le dichlorvos, le malathion et le fénitrothion et la terre Tonsil 371 pour la deltaméthrine. Le chlorpyriphos-méthyl est, quant à lui, plus efficacement éliminé avec la terre Tonsil EX 941 contenant $10 \%$ de charbon actif (CA), ce qui laisse supposer que le chlorpyriphos-méthyl serait sensible au CA contrairement aux quatre autres pesticides précédemment cités. Quant au pyrimiphos-méthyl, il est, dans ces essais, totalement éliminé quelle que soit la terre utilisée. Ce comportement tout à fait exceptionnel est probablement dû à la présence du doublet électronique de l'atome d'azote de la molécule qui lui confère une polarité intéressante en l'occurrence. Malgré l'efficacité de certaines terres à éliminer des résidus de pesticides, les teneurs résiduelles restent dans la plupart des cas supérieures aux LMR fixées pour les graines oléagineuses. Dans les essais réalisés, les taux d'élimination obtenus avec la terre la plus efficace varient pour chaque pesticide étudié : environ $50 \%$ pour le dichlorvos, $30 \%$ pour le malathion, $30 \%$ pour la deltaméthrine, de 20

\footnotetext{
${ }^{8}$ Raffinage chimique seul.

${ }^{9}$ Le $\mathrm{K}_{\mathrm{O} / \mathrm{E}}(\log \mathrm{P})$ est un critère reconnu par les experts de l'étude des produits antiparasitaires; pour les pesticides, il indique la probabilité du transfert d'une substance d'un milieu (sol, eau) à des organismes, et il est souvent utilisé pour le calcul du facteur de bioconcentration (FBC), indice du risque de bioaccumulation.
} 
Tableau 1. Conditions des opérations de raffinage par voie chimique ou par distillation neutralisante (raffinage " physique ») appliquées dans l'étude de l'élimination des résidus de pesticides. D'après Pagès et al. (2004, 2006).

\begin{tabular}{|c|c|c|c|}
\hline \multicolumn{2}{|l|}{ Raffinage chimique } & \multicolumn{2}{|l|}{ Raffinage " physique " } \\
\hline Étapes & $\begin{array}{l}\text { Conditions selon } \\
\text { capacités équipements } \\
\text { pilote ( } 25 \mathrm{~kg} \text { ou } 1 \mathrm{t})\end{array}$ & Étapes & $\begin{array}{l}\text { Conditions selon } \\
\text { capacités équipements } \\
\text { pilote }(25 \mathrm{~kg} \text { ou } 1 \mathrm{t})\end{array}$ \\
\hline $\begin{array}{l}\text { Dégommage } \\
\text { Conditionnement acide }\end{array}$ & $\begin{array}{l}3 \% \text { d'eau } \\
70{ }^{\circ} \mathrm{C}, 30 \text { min } \\
0,1 \% \text { acide } \\
\text { phosphorique } \\
60 \text { ou } 70^{\circ} \mathrm{C}, 45 \mathrm{~min}\end{array}$ & Prétraitement & $\begin{array}{l}0,1 \% \text { acide } \\
\text { phosphorique, } \\
(1 \% \text { d'eau) } \\
60 \text { ou } 70^{\circ} \mathrm{C}, 45 \mathrm{~min}\end{array}$ \\
\hline Neutralisation & $\begin{array}{l}\text { Soude } 3 \mathrm{~N} \text { (ou } 1 \mathrm{~N}) \\
70 \text { ou } 75^{\circ} \mathrm{C}\left(80^{\circ} \mathrm{C}\right) \text {, } \\
30 \text { min }\end{array}$ & & \\
\hline $\begin{array}{l}\text { Centrifugation, } \\
\text { lavages, séchage }\end{array}$ & & $\begin{array}{l}\text { Centrifugation } \\
\text { séchage }\end{array}$ & \\
\hline Décoloration & $\begin{array}{l}0,5-1 \% \text { de terres } \\
90{ }^{\circ} \mathrm{C}, 40 \text { mbar, } \\
40-45 \text { min }\end{array}$ & Décoloration & $\begin{array}{l}1 \% \text { de terres } \\
90{ }^{\circ} \mathrm{C}, 40 \mathrm{mbar}, \\
40-45 \mathrm{~min}\end{array}$ \\
\hline Filtration & & Filtration & \\
\hline Désodorisation & $\begin{array}{l}\text { Essais } 25 \mathrm{~kg} \text { : } \\
180^{\circ} \mathrm{C} 6 \mathrm{~h} \text { et } 220{ }^{\circ} \mathrm{C} 4 \mathrm{~h} \text {, } \\
0,5 \text { et } 1,5 \% / \mathrm{h} \text { de vapeur, } \\
3 \mathrm{mbar} \\
\text { Essais } 1 \mathrm{t} \text { : } \\
180^{\circ} \mathrm{C}, 2 \mathrm{~h} 30 \text { ou } 220^{\circ} \mathrm{C} \text {, } \\
3 \mathrm{~h}, 0,5 \% / \mathrm{h} \text { de vapeur, } \\
3 \mathrm{mbar} \\
220^{\circ} \mathrm{C}, 1-4 \mathrm{~h}, 1 \% / \mathrm{h} \text { de } \\
\text { vapeur, } 3-4 \mathrm{mbar}\end{array}$ & Distillation neutralisante & $\begin{array}{l}\text { Essais } 25 \mathrm{~kg} \text { : } \\
240{ }^{\circ} \mathrm{C}, 3 \mathrm{~h}, 0,5 \text { et } 1,5 \% / \mathrm{h} \\
\text { de vapeur, } 3 \mathrm{mbar} \\
\text { Essais } 1 \mathrm{t} \text { : } \\
240{ }^{\circ} \mathrm{C}, 2 \mathrm{~h} 30-4 \mathrm{~h}, 1 \% / \mathrm{h} \\
\text { de vapeur, } 4 \mathrm{mbar}\end{array}$ \\
\hline
\end{tabular}

à $25 \%$ pour le fénitrothion et $10 \%$ pour le chlorpyriphos-méthyl. Cet ordre d'efficacité rappelle (sauf pour la deltaméthrine) le classement basé sur la solubilité dans l'eau des molécules actives; le caractère hydrophile d'un pesticide influence donc probablement son élimination à la décoloration où les terres retiennent, outre les pigments, des molécules amphiphiles (phospholipides et savons résiduels), ceci corroborant l'hypothèse du rôle des sites cationiques des molécules éliminées.

\section{Désodorisation et distillation neutralisante}

C'est très souvent l'étape déterminante pour l'élimination des résidus de pesticides. Les paramètres cruciaux de cette étape sont la température, la durée et la quantité de vapeur injectée. L'augmentation de la durée est surtout importante pour les résidus rémanents et présents en quantité plus importante; l'accroissement de la température augmente le taux de vaporisation des molécules de pesticides, favorisant ainsi leur entraînement par la vapeur et son impact est plus sensible pour les molécules les plus lourdes (critère poids moléculaire) ; le débit de vapeur d'eau injectée

contenus dans les huiles brutes. Chaque type de raffinage présente cependant ses spécificités et avantages. Le raffinage chimique permet, selon la nature des molécules, de diminuer leurs teneurs résiduaires avant l'étape de désodorisation, alors que le raffinage "physique » présente l'avantage d'opérer à une température plus élevée à la distillation neutralisante, condition favorisant leur élimination. Même avec les niveaux de dopage les plus élevés, rarement rencontrés dans la réalité, les huiles raffinées obtenues ont toutes des teneurs résiduelles inférieures aux LMR fixées dans les oléagineux, exception faite pour la deltaméthrine qui, avec l'endosulfan, sont les seuls pesticides pouvant présenter un risque réel du fait de leur élimination difficile qui interdit toute contamination importante d'une huile brute sous peine de retrouver des résidus en quantité non négligeable dans I'huile raffinée.

Des travaux similaires concernant le raffinage des huiles d'olive publiés par Ruiz-mendez et al. (2005) confirment les résultats exposés plus haut, notamment pour l'endosulfan.

L'élimination des résidus de pesticides présents dans des huiles brutes industrielles (non dopées) par raffinage chimique sur sites industriels est documentée par Van Duijn (2008) dans le cadre d'une étude intégrant :

a) les pratiques en amont (emploi des pesticides dans la chaîne d'approvisionnement en oléagineux);

b) l'évaluation des facteurs de concentration en trituration ;

c) I'analyse des résidus dans les huiles brutes ;

d) un essai industriel d'élimination de résidus phytosanitaires ;

e) la collecte des résultats de validation du procédé de raffinage pour différents pesticides ${ }^{10}$ présents dans des huiles brutes de tournesol, de colza et de soja identifiées en phase c).

Avec les procédés mis en œuvre sur les sites de raffinage industriel de l'étude, tous les pesticides résiduels des huiles brutes traitées ont été ramenés à des teneurs inférieures aux limites de quantification. Par ailleurs, les résultats de l'essai industriel relatifs au comportement des différents pesticides à chaque étape du raffinage (phase d) vont dans le même sens que ceux obtenus à l'échelle pilote sur huiles brutes dopées exposés plus haut ou à l'échelle du laboratoire (références citées par Van Duijn, 2008).

\section{Élimination des résidus d'hydrocarbures polycycliques aromatiques (HAP)}

La nature, l'origine, les niveaux d'occurrence et le statut réglementaire de ce type de contaminant sont rappelés dans ce dossier par l'article de Lacoste et al. ${ }^{11}$.

\footnotetext{
${ }^{10}$ Fénitrothion, malathion, pirimiphos-méthyl, parathion-éthyl, dichlorvos, chlorpyriphos, chlorpyriphos-méthyl et endosulfan total.

11 « Détection de contaminants dans les huiles végétales : bilan à fin 2009 ».
} 
Tableau 2. Comportement aux différentes étapes du raffinage (chimique et "physique ») de quelques pesticides et leurs caractéristiques physicochimiques d'intérêt (conditions dans le tableau 1). D'après Pagès et al. (2006).

\begin{tabular}{|c|c|c|c|c|c|c|}
\hline \multirow[b]{2}{*}{ Catégories } & \multirow[b]{2}{*}{ Pesticide } & \multicolumn{3}{|l|}{ Étapes du raffinage (1) } & \multicolumn{2}{|c|}{$\begin{array}{l}\text { Caractéristiques physicochimiques } \\
\text { d'intérêt }\end{array}$} \\
\hline & & Conditionnement acide & $\begin{array}{l}\text { Neutralisation } \\
\text { (raffinage } \\
\text { chimique seul) }\end{array}$ & $\begin{array}{l}\text { Lavages } \\
\text { (raffinage } \\
\text { chimique seul) }\end{array}$ & $\begin{array}{l}K_{0 / E} \\
(\log P)\end{array}$ & $\begin{array}{l}\text { Solubilité dans } \\
\text { I'eau (mg/L) }\end{array}$ \\
\hline Organophosphoré & Dichlorvos & & +++ & +++ & 1,47 & 8800 \\
\hline Organophosphoré & Malathion & & ++ & ++ & 2,89 & 145 \\
\hline Organophosphoré & Fénitrothion & & & & 3,38 & 14 \\
\hline Organophosphoré & Pirimiphos-méthyl & + & & & 4,2 & 5 \\
\hline Organophosphoré & Chlorpyriphos-méthyl & & + & & 4,2 & 4 \\
\hline Organochlorés & $\alpha+\beta$ endosulfan ${ }^{a}$ & & & & 3,6 & 0,32 \\
\hline Pyréthroïde & Deltaméthrine & & + & & 4,6 & 0,002 \\
\hline \multicolumn{5}{|c|}{ Étapes du raffinage (2) } & \multicolumn{2}{|c|}{$\begin{array}{l}\text { Caractéristiques physicochimiques } \\
\text { d'intérêt }\end{array}$} \\
\hline Catégories & Pesticide & \multicolumn{2}{|c|}{ Décoloration } & $\begin{array}{l}\text { Désodorisation } \\
\text { ou distillation } \\
\text { neutralisante }\end{array}$ & $\begin{array}{l}\text { Poids } \\
\text { moléculaire } \\
(\mathrm{g} / \mathrm{mol})\end{array}$ & $\begin{array}{l}\text { Tension de } \\
\text { vapeur } \\
(\mathrm{mPa})\end{array}$ \\
\hline Organophosphoré & Dichlorvos & \multicolumn{2}{|l|}{ ++ } & +++ & 221 & 1600 \\
\hline Organophosphoré & Malathion & \multicolumn{2}{|l|}{+} & $+++(V S)$ & 330 & 5,3 \\
\hline Organophosphoré & Fénitrothion & \multicolumn{2}{|l|}{+} & +++ (VS) & 277 & 18 \\
\hline Organophosphoré & Pirimiphos-méthyl & \multicolumn{2}{|l|}{+++} & +++ & 305 & 2 \\
\hline Organophosphoré & Chlorpyriphos-méthyl & & & +++ (VS) & 323 & 2,4 \\
\hline Organochlorés & $\alpha+\beta$ endosulfan ${ }^{a}$ & & & $++($ VS) & 407 & 0,83 \\
\hline Pyréthroïde & Deltaméthrine & + & & ++ & 505 & 0,012 \\
\hline
\end{tabular}

+++ : Étape très efficace; VS : quantité de vapeur suffisante.

${ }^{a}$ Caractéristiques physicochimiques données pour le mélange $2 / 3 \alpha+1 / 3 \beta$ endosulfan.

Des travaux réalisés en 2004, 2005 et 2009 par I'ITERG sur ses installations pilote ont étudié l'élimination de quatre HAP (tableau 3) par raffinage chimique ou par distillation neutralisante (raffinage "physique ») d'huiles végétales dopées à différents niveaux (600 et $2000 \mu \mathrm{g} / \mathrm{kg}$ ).

\section{Conditionnement acide, neutralisation alcaline, lavages et séchage}

Ces étapes réduisent peu (de l'ordre de $20 \%$ pour un dopage de $600 \mu \mathrm{g} / \mathrm{kg}$ et de $5 \%$ seulement pour le dopage à $2000 \mu \mathrm{g} / \mathrm{kg}$ ) la quantité des quatre HAP étudiés. L'inefficacité de ces étapes pourrait être similaire à celle rencontrée pour l'élimination de certains pesticides: solubilité dans I'eau quasi nulle et $\mathrm{K}_{\mathrm{O} / \mathrm{E}}(\log \mathrm{P})$ révélant un fort caractère lipophile (tableau 3).
Décoloration (1 \% de terre décolorante avec 0,5 ou $1 \%$ de $(A)$

Cette étape, à condition qu'elle soit réalisée en présence de CA, est connue pour son efficacité sur l'élimination des HAP lourds. Plusieurs types de charbon ont été testés ${ }^{12}$, et la sélection d'un CA se révèle importante en termes d'efficacité (figure 3): un charbon activé physiquement est plus efficace qu'un charbon activé chimiquement; les caractéristiques du CA (surface spécifique, granulométrie, diamètre des pores) et ses propriétés de filtrabilité après décoloration (risque de colmatage des filtres) sont des paramètres également importants à prendre en compte. La quantité de CA utilisé

\footnotetext{
${ }^{12}$ CAL TR et CAL I (fournisseur Chemviron Carbon), Acticarbone 3S - devenu 3SW (fournisseur CECA).
}

influe sur le taux de contaminants éliminés ; la limite pratique du taux d'emploi est la filtrabilité et le coût. L'étude de la durée de l'étape de décoloration (figure 3) a révélé que, pour atteindre une teneur résiduelle inférieure à la teneur maximale réglementaire de $2 \mu \mathrm{g} / \mathrm{kg}$, l'adsorption des HAP lourds sur le CA doit être suffisamment prolongée (45 minutes dans les essais sur huile dopée à $600 \mu \mathrm{g} / \mathrm{kg}$ ) même si assez rapidement (dès 15 minutes) le traitement se montre efficace (mais insuffisant). La température de décoloration peut influencer de manière positive l'élimination des HAP; bien que faible, ce gain peut parfois permettre $d^{\prime}$ atteindre le seuil fixé par la réglementation $(2 \mu \mathrm{g} / \mathrm{kg}$ en $B(a) P)$.

Dans le cas d'une contamination importante (niveau $2000 \mu \mathrm{g} / \mathrm{kg}$ ), l'étape de décoloration avec CA se révèle également très efficace, et il 
Tableau 3. Composés retenus pour l'étude de l'élimination des HAP au raffinage et caractéristiques physicochimiques d'intérêt. Travaux ITERG 2005 (non publiés).

\begin{tabular}{|c|c|c|c|c|c|c|c|}
\hline HAP & $\mathbf{N}^{\circ}{ }^{\circ}$ des cas & Type & $\begin{array}{c}\text { Poids } \\
\text { moléculaire } \\
(\mathrm{g} / \mathrm{mol})\end{array}$ & $\begin{array}{c}\begin{array}{c}\text { Solubilité } \\
\text { dans I'eau } \\
\left(\mathrm{mg} / \mathrm{L} \text { à } 25^{\circ} \mathrm{C}\right)\end{array}\end{array}$ & $\begin{array}{c}K_{\mathbf{O} / E} \\
(\log P)\end{array}$ & $\begin{array}{l}\text { Pt ébullition } \\
\left({ }^{\circ} \mathrm{C} \text { à } \mathbf{P}_{\mathrm{atm}}\right)\end{array}$ & $\begin{array}{c}\text { Tension de } \\
\text { vapeur } \\
\left(\mathrm{Pa} \text { à } 20^{\circ} \mathrm{C}\right)\end{array}$ \\
\hline Anthracène & $120-12-7$ & \multirow{2}{*}{$\begin{array}{c}\text { Léger } \\
3 \text { noyaux aromatiques }\end{array}$} & 178,23 & 1,29 & 4,45 & 340 & $9,1 \times 10^{-2}$ \\
\hline Phénanthrène & $85-01-8$ & & 178,23 & 1,2 & 4,57 & 341 & $3,6 \times 10^{-4}$ \\
\hline Benzo(a)pyrène & $50-32-8$ & \multirow{2}{*}{$\begin{array}{l}\text { Lourd } \\
5 \text { noyaux aromatiques }\end{array}$} & 252,31 & 0,0031 & 6 & 475 & $7,3 \times 10^{-7}$ \\
\hline Dibenz(a,h)anthracène & $53-70-3$ & & 278,35 & 0,0005 & 6,7 & 524 & $1,3 \times 10^{-8}$ \\
\hline
\end{tabular}

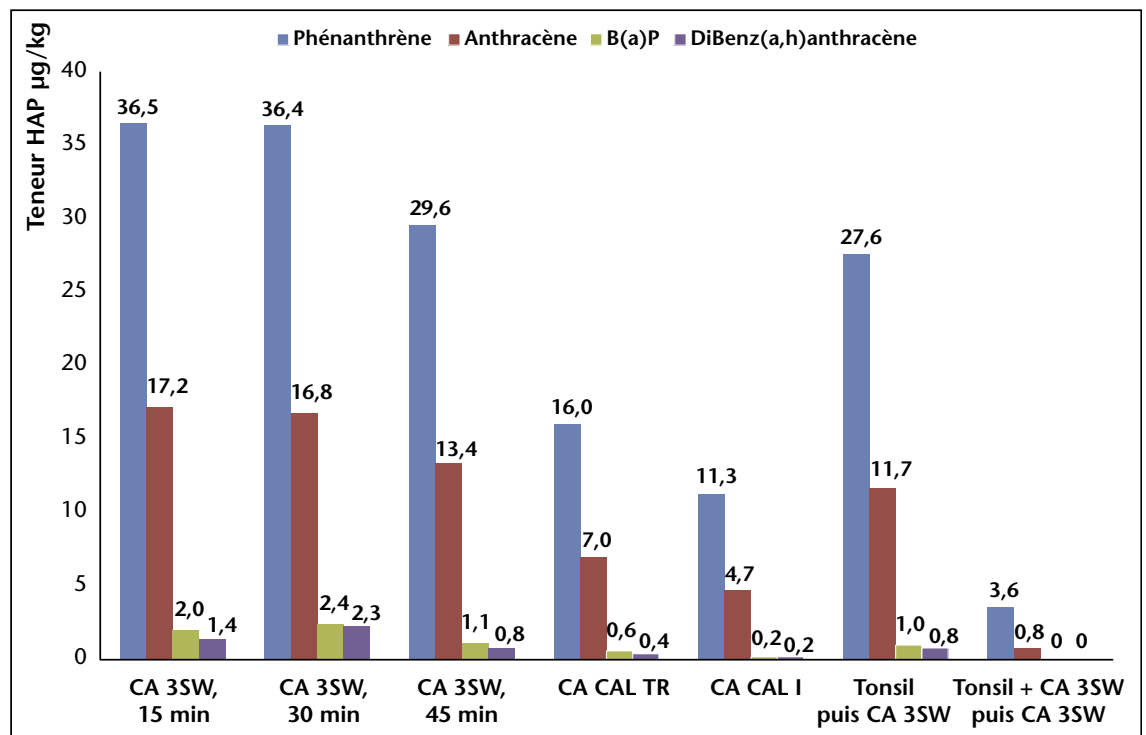

Figure 3. Élimination des HAP à la décoloration $(0,5 \%$ de terre décolorante $+0,5 \%$ de charbon actif [CA]) à $90^{\circ} \mathrm{C}, 45$ minutes - Influence de la durée $(15,30$ et 45 minutes), de la nature du CA (Acticarbone 3SW, CAL TR, CAL I) et d'un traitement séquentiel terre/CA. Teneurs en HAP de I'huile conditionnée, neutralisée, lavée, séchée avant décoloration: Phé., $665 \mu \mathrm{g} / \mathrm{kg}$; Anthr., $612 \mu \mathrm{g} / \mathrm{kg}, \mathrm{B}(\mathrm{a}) \mathrm{P}, 637 \mu \mathrm{g} / \mathrm{kg}$ et Dibenz(a,h)anthr., $776 \mu \mathrm{g} / \mathrm{kg}$. Travaux ITERG 2004, 2005.

n'est pas nécessaire de répéter cette étape pour atteindre des teneurs résiduelles en HAP $(B(a) P)$ conformes à la réglementation. Étude de l'efficacité d'un traitement séquentiel : deux options de séquençage ont été testées; soit séparer la mise en œuvre des terres décolorantes (Tonsil) et du CA (Acticarbone 3SW), soit traitement couplé terres + CA (Tonsil $+3 S W$ ) suivi d'un traitement par terre de décoloration seule (3SW). Si la première option permet une légère amélioration de l'élimination des HAP, le gain n'est cependant pas suffisant pour envisager le développement de cette technique; la seconde option est beaucoup plus efficace, davantage même qu'avec le CA ayant donné les meilleurs résultats en traitement classique (couplé aux terres): élimination totale des HAP lourds ( $B(a) P$ et dibenz(a,h)anthracène) et teneurs résiduelles en HAP légers, phénanthrène et anthracène, significativement diminuées (figure 3).
Emploi de plaques filtrantes chargées en $C A$ : de très récents essais ont permis de tester l'efficacité de plaques filtrantes chargées en CA. Une huile de colza raffinée, simplement dopée à $10 \mu \mathrm{g} / \mathrm{kg}$ de $\mathrm{B}(\mathrm{a}) \mathrm{P}$, chauffée à $80^{\circ} \mathrm{C}$ sous agitation, a été filtrée sur filtre-presse muni de plaques (référence AMAFILTER PFCAS3TC 20*20 Eurofiltec) garnies de CA standard Eurofiltec DARCO S51; le taux de CA dans une plaque est de $65 \%$ (par rapport au poids de la plaque), soit environ $32,5 \mathrm{~g}$ par plaque ; les caractéristiques physiques de ces plaques sont données dans le tableau 4. Le temps de contact entre I'huile et le CA pouvant être un point critique et limitant, plusieurs filtrations ont été réalisées, mais les résultats obtenus sont très encourageants, avec une élimination presque totale du $\mathrm{B}(\mathrm{a}) \mathrm{P}$ dès la première filtration et une résiduelle inférieure à $0,1 \mu \mathrm{g} / \mathrm{kg}$ après cinq passages. Une validation à l'échelle semi-industrielle est prévue pour confirmer ces résultats.

\section{Désodorisation et distillation neutralisante}

Pour mieux mettre en évidence les effets de la désodorisation sur l'élimination des HAP légers, un essai a été conduit sur une huile dopée ayant subi les premières étapes d'un raffinage chimique et une décoloration sans CA afin de maintenir un niveau de contamination élevé avant la désodorisation; le résultat confirme que la désodorisation n'a pas d'effet sur l'élimination des HAP lourds quelles que soient les conditions appliquées (température, quantité de vapeur); en revanche, cette étape est efficace pour l'élimination des HAP légers qui augmente avec la température (optimale à $220^{\circ} \mathrm{C}$ ) (figure 4). Les essais de raffinage complet (et décoloration avec CA) par voie chimique ou par distillation neutralisante (raffinage "physique », figure 5) d'une huile dopée à environ $600 \mu \mathrm{g} / \mathrm{kg}$ ont conduit à des huiles raffinées présentant de très faibles concentrations résiduelles en HAP, conforme à la limite réglementaire $(2 \mu \mathrm{g} / \mathrm{kg}$ en $\mathrm{B}(\mathrm{a}) \mathrm{P})$.

\section{Conclusion}

Les étapes de décoloration (terre et CA) et de désodorisation sont essentielles et complémentaires dans l'élimination des HAP. II est vivement recommandé de valider le choix d'un CA par des essais préliminaires.

\section{Élimination d'autres contaminants}

\section{Traces de solvants d'extraction (hexane)}

La teneur maximale en hexane résiduel est fixée à $1 \mathrm{mg} / \mathrm{kg}$ par la directive 2009/32/CE ${ }^{13}$ concernant les solvants d'extraction utilisés dans la fabrication des denrées alimentaires et de leurs ingrédients.

\footnotetext{
${ }^{13}$ Relative au rapprochement des législations des États membres : refonte de la directive 88/344/CEE modifiée.
} 
Tableau 4. Caractéristiques physiques des plaques chargées en charbon actif (référence AMAFILTER PFCAS3TC 20*20 Eurofiltec). Essais ITERG, 2009.

\begin{tabular}{|c|c|c|c|c|c|c|}
\hline \multicolumn{7}{|c|}{ Caractéristiques physiques } \\
\hline Référence plaques & Épaisseur (mm) & Grammage $\left(\mathrm{gr} / \mathrm{m}^{2}\right)$ & $\begin{array}{l}V f^{a} \\
\left(L / m^{2} \text { par minute) }\right.\end{array}$ & Es $^{\mathbf{b}}$ (bars) & Eh' (bars) $^{c}$ & Taux de CA (\%) \\
\hline $3 T C$ & $4,4-4,8$ & $1250-1350$ & $62-133$ & 1 & 0,4 & 65 \\
\hline
\end{tabular}

a Perméabilité - Fluide : eau déminéralisée - spécification Eurofiltec $n^{\circ} 10498.0$.

${ }^{b}$ Pression d'éclatement à sec - spécification Eurofiltec $n^{\circ} 10498.04$.

' Pression d'éclatement humide - spécification Eurofiltec n ${ }^{\circ} 10498.04$.

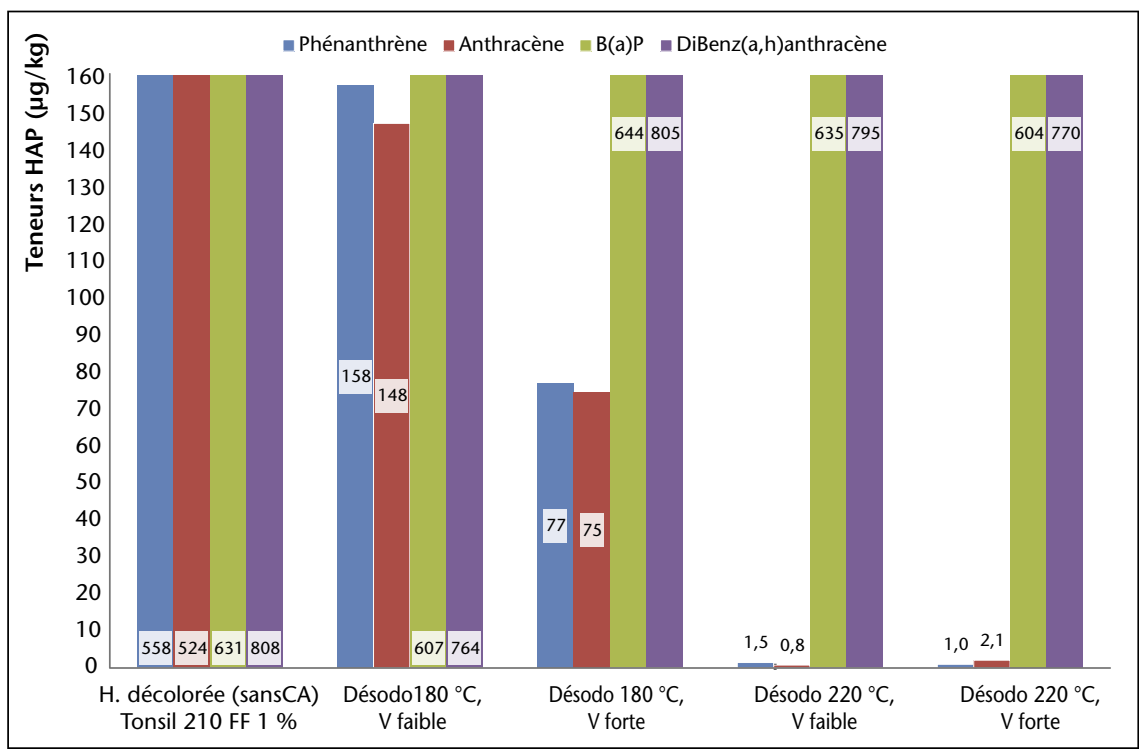

Figure 4. Élimination des HAP légers à la désodorisation d'une huile dopée ayant subi les premières étapes d'un raffinage chimique et décolorée sans charbon actif - Influence de la température et de la quantité de vapeur injectée; durée : trois heures. Travaux ITERG 2004, 2005.

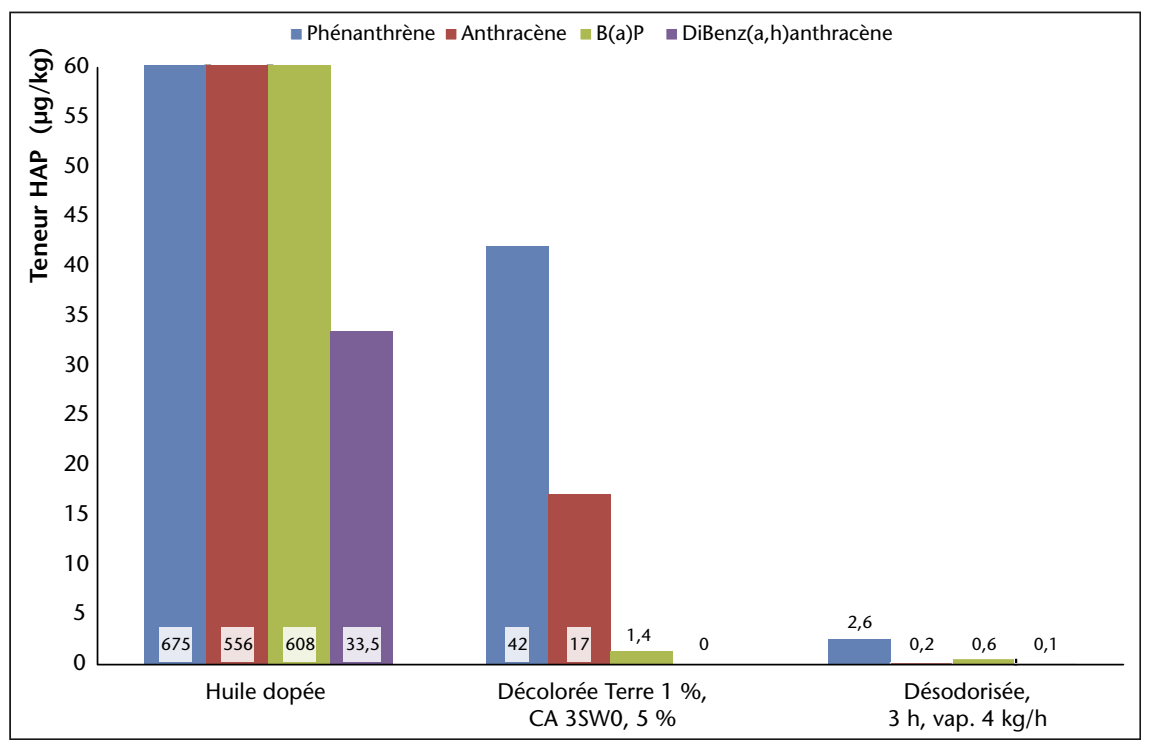

Figure 5. Évolution de la teneur en HAP au cours d'un raffinage par distillation neutralisante ("physique »). Travaux ITERG 2004, 2005.

Les traces résiduelles d'hexane dans les huiles brutes sont éliminées au cours du raffinage à l'étape de décoloration qui est réalisée sous

vide ainsi qu'à l'étape de désodorisation dont les conditions $\left(\mathrm{T}>20{ }^{\circ} \mathrm{C}\right.$, vide poussé, stripping à la vapeur d'eau) garantissent l'absence de traces de solvant après raffinage, ce qui est vérifié par les contrôles de conformité des huiles raffinées.

\section{Traces métalliques}

Fer, cuivre : spécifications et bonnes pratiques de fabrication (BPF)

Les normes commerciales du Codex Alimentarius recommandent des teneurs limites en fer et cuivre pour les huiles et graisses végétales raffinées: respectivement 1,5 et $0,1 \mathrm{mg} / \mathrm{kg}$. Mais les BPF vont au-delà de ces recommandations : fer inférieur à $0,1-0,2 \mathrm{mg} / \mathrm{kg}$ et cuivre inférieur à 0,02-0,05 mg/kg.

\section{Autres éléments métalliques : \\ spécifications réglementaires}

- Le règlement (CE) n 1881/2006 modifié fixe (annexe, section 3 ) des teneurs limites en plomb $(0,1 \mathrm{mg} / \mathrm{kg})$ dans les huiles et matières grasses, et en cadmium $(0,2 \mathrm{mg} / \mathrm{kg})$ dans les graines de soja. Précisons que la présence éventuelle de ces deux éléments n'est pas liée aux pratiques culturales, mais à la pollution de l'environnement;

- les teneurs maximales en résidus de catalyseurs sont fixées en France par l'arrêté du 19 octobre $2006^{14}$ modifié, relatif à l'emploi d'auxiliaires technologiques dans la fabrication de certaines denrées alimentaires. Parmi les catalyseurs d'hydrogénation, le nickel est le plus courant, et sa dose résiduelle maximale est fixée à 0,2 mg/kg.

\section{Élimination des traces métalliques au raffinage}

Les traces métalliques sont classiquement éliminées, au cours du raffinage, en partie à l'étape de neutralisation chimique et surtout lors de la décoloration ou en prétraitement avec un agent d'adsorption lors du raffinage physique. L'efficacité de l'étape d'adsorption dépend de la nature de l'adsorbant employé : terres décolorantes et/ou trysil (silice) s'avèrent très efficaces.

\section{Contrôle des niveaux de traces métalliques dans les huiles raffinées}

L'occurrence de résidus de traces métalliques dans les corps gras raffinés a fait l'objet de

${ }^{14}$ JORF du 2 décembre 2006. 
plusieurs études publiées, déjà anciennes et résumées par Castera (1992) : les teneurs en fer et en cuivre restent respectivement inférieures ou égales à $0,1 \mathrm{mg} / \mathrm{kg}$ (ou $100 \mu \mathrm{g} / \mathrm{kg}$ ) et inférieures ou égales à $0,03 \mathrm{mg} / \mathrm{kg}$ (ou $30 \mu \mathrm{g} / \mathrm{kg}$ ) pour les valeurs les plus hautes dans la majorité des huiles de colza, de tournesol et de soja raffinées analysées, reflétant les BPF citées plus haut; les teneurs en cadmium et en plomb sont respectivement inférieures à $0,005 \mathrm{mg} / \mathrm{kg}$ et comprises entre 0,01 et 0,02 $\mathrm{mg} / \mathrm{kg}$ dans les huiles raffinées, ces valeurs étant proches des limites de quantification de la méthode d'analyse et nettement inférieures aux spécifications réglementaires citées ci-dessus.

Plus récemment, Dugo et al. (2004) font état de la contamination en plomb et en cadmium $d$ 'huiles végétales raffinées $d$ 'arachide, de tournesol, de soja et de pépins de raisin (tableau 5). Les teneurs décelées confirment le très faible niveau de contamination des huiles raffinées en ces deux éléments et les résultats d'enquêtes réalisées par I'ITERG entre 1992 et 1999 (non publiés) : $100 \%$ des teneurs déterminées dans 42 huiles végétales raffinées prélevées en France n'atteignaient pas la limite réglementaire de $0,1 \mathrm{mg} / \mathrm{kg}$ pour le plomb
( $<0,02 \mathrm{mg} / \mathrm{kg}$, limite de quantification) et pour le cadmium, étaient toutes inférieures ou égales à $0,002 \mathrm{mg} / \mathrm{kg}$ (limite de quantification).

\section{Aflatoxines}

Rappelons que la contamination par les aflatoxines est liée au développement de moisissures (Aspergillus) dans les graines oléagineuses quand elles sont placées dans des conditions particulières de température $\left(28\right.$ à $\left.33^{\circ} \mathrm{C}\right)$ et $d^{\prime}$ humidité $\left(A_{W}>0,8\right)$. Les aflatoxines $d^{\prime}$ Aspergillus flavus sont particulièrement toxiques et cancérigènes. Leur structure chimique les rend peu solubles dans les huiles: leur contamination est donc généralement cinq fois plus faible que dans les graines dont elles sont extraites (quand elles sont contaminées).

L'annexe, section 2 du règlement (CE) $\mathrm{n}^{\circ} 1881 / 2006$ du 19 décembre 2006, fixe les teneurs maximales en aflatoxines dans les arachides, les fruits à coques et tous produits dérivés, tels que les huiles, destinés à la consommation directe ou à l'emploi en tant qu'ingrédient alimentaire (tableau 6).
Il découle de cette réglementation européenne que la teneur maximale en aflatoxine $B_{1}$ dans les huiles (vierges ou raffinées) d'arachide, de noix, de noisette, d'amande, de pistache ou de tout autres fruits à coque est fixée à $2 \mu \mathrm{g} / \mathrm{kg}$. Pour toutes les autres huiles (de graines ou de fruits tels que l'olive, éventuellement) vierges ou en mélange avec des huiles raffinées, I'administration française (DGCCRF, 1990) continue à prendre pour référence l'avis du Conseil supérieur d'hygiène publique de France, qui fixe la limite en aflatoxine $B_{1}$ à $5 \mu \mathrm{g} / \mathrm{kg}$.

\section{Élimination des mycotoxines au raffinage}

Quelle que soit leur concentration, les aflatoxines sont éliminées au cours des étapes d'un raffinage chimique classique: l'action de la soude à la neutralisation en détruit 90 à $98 \%$; le résidu est éliminé à la décoloration (Denise, 1983). Les contrôles concernent surtout les huiles vierges.

\section{Qualité microbiologique}

Aucune multiplication microbienne n'étant possible en absence d'eau, les huiles et graisses végétales anhydres ne posent aucun problème de stabilité microbiologique (Jouve, 1993).

Tableau 5. État de la contamination en plomb et cadmium des huiles végétales (Dugo et al., 2004).

\begin{tabular}{|c|c|c|c|c|c|c|c|}
\hline \multirow[t]{2}{*}{ Huiles } & \multirow{2}{*}{$\begin{array}{l}\text { Nombre } \\
\text { d'échantillons }\end{array}$} & \multicolumn{3}{|c|}{ Plomb $(\mu \mathrm{g} / \mathbf{k g})$} & \multicolumn{3}{|c|}{ Cadmium $(\mu \mathrm{g} / \mathbf{k g})$} \\
\hline & & Minimum & Maximum & Moyenne & Minimum & Maximum & Moyenne \\
\hline Arachide & 6 & 5,1 & 15,6 & 10,0 & $<0,5$ & 3,5 & 2,2 \\
\hline Tournesol & 5 & 5,0 & 15,5 & 10,0 & 0,5 & 4,1 & 1,7 \\
\hline Soja & 5 & 10,7 & 43,3 & 25,5 & 2,5 & 5,0 & 3,7 \\
\hline Pépins de raisin & 3 & 7,5 & 15,6 & 11,4 & 2,1 & 4,6 & 3,6 \\
\hline
\end{tabular}

Tableau 6. Extrait de l'annexe, section 2 (mycotoxines), du règlement CE $n^{\circ}$ 1881/2006 concernant les aflatoxines et la zéaralénone dans les huiles végétales.

\begin{tabular}{|c|c|c|c|c|}
\hline & \multirow[t]{2}{*}{ Mycotoxine/denrées alimentaires } & \multicolumn{3}{|c|}{ Teneurs maximales $(\mu \mathrm{g} / \mathbf{k g})$} \\
\hline & & $\overline{\mathbf{B}_{1}}$ & Somme $B_{1}, B_{2}, G_{1}+G_{2}$ & $M_{1}$ \\
\hline 2.1. & Aflatoxines & & & \\
\hline 2.1 .1 & $\begin{array}{l}\text { Arachides destinées à être soumises à un traitement de tri ou à d'autres méthodes physiques } \\
\text { avant consommation humaine ou utilisation comme ingrédient de denrées alimentaires }\end{array}$ & $8^{a}$ & $15^{\mathrm{a}}$ & - \\
\hline 2.1 .2 & $\begin{array}{l}\text { Fruits à coque destinés à être soumis à un traitement de tri ou à d'autres méthodes physiques } \\
\text { avant consommation humaine ou utilisation comme ingrédient de denrées alimentaires }\end{array}$ & $5^{\mathrm{a}}$ & $10^{\mathrm{a}}$ & - \\
\hline 2.1 .3 & $\begin{array}{l}\text { Arachides, fruits à coques et produits dérivés de leur transformation, destinés à la consommation } \\
\text { humaine directe ou à une utilisation comme ingrédient de denrées alimentaires }\end{array}$ & $2^{\mathrm{a}}$ & $4^{\mathrm{a}}$ & - \\
\hline 2.5 & Zéaralénone & \multicolumn{3}{|c|}{ Teneur maximale $(\mu \mathbf{g} / \mathbf{k g})$} \\
\hline 2.5 .4 & Huile de maïs raffinée & \multicolumn{3}{|c|}{$400^{b}$} \\
\hline
\end{tabular}

${ }^{a}$ Les teneurs maximales se réfèrent à la partie des arachides et fruits à coques destinée à être consommée. Si les arachides et fruits à coques « entiers » sont analysés, on suppose, lors du calcul de la teneur en aflatoxines, que toute la contamination se trouve sur la partie destinée à être consommée.

${ }^{\mathrm{b}}$ Teneur maximale applicable depuis le $1^{\mathrm{er}}$ octobre 2007. 
Tableau 7. Qualité microbiologique : exemples de spécifications respectées dans les huiles végétales.

\begin{tabular}{|lll|}
\hline Analyse & Méthode de référence & Exemple de spécifications \\
\hline Salmonelles & Afnor TEC-24-2-04/03 & Absence $/ 25 \mathrm{~g}$ \\
\hline Levures & NF V 08-059 (2002) & $<1 / \mathrm{g}$ \\
\hline Moisissures & NF V 08-059 (2002) & $<1 / \mathrm{g}$ \\
\hline Micro-organismes aérobies $30^{\circ} \mathrm{C}$ & NF V 08-051 (1999) & $<10 / \mathrm{g}$ \\
\hline Bacillus cereus & XP V 08-058 (1995) & $<10 / \mathrm{g}$ \\
\hline Escherichia coli & NF V 08-053 (2002) & $<1 / \mathrm{g}$ \\
\hline Coliformes thermotolérants & NF V 08-060 (1996) & $<1 / \mathrm{g}$ \\
\hline Coliformes $30^{\circ} \mathrm{C}$ & NF V 08-050 (1999) & $<1 / \mathrm{g}$ \\
\hline
\end{tabular}

C'est tout particulièrement le cas des huiles raffinées pour lesquelles le traitement de désodorisation constitue un traitement stérilisant très efficace permettant $d^{\prime}$ obtenir des huiles de qualité microbiologique irréprochable et conforme, par exemple, aux données du tableau 7.

\section{Résidus de dioxines et de polychlorobiphényles (PCB)}

Les dioxines sont un ensemble formé de 75 congénères de la famille des dibenzop-dioxines polychlorées (PCDD) et de 135 congénères de la famille des polychlorodibenzofuranes (PCDF). Les PCB constituent un groupe de 209 congénères dont 12 présentent des propriétés toxicologiques analogues à celles des dioxines et sont donc souvent qualifiés de «PCB de type dioxine ».

L'annexe, section 5 du règlement (CE) $n^{\circ} 1881 / 2006$, fixe des teneurs maximales en picogramme par gramme pour les huiles et graisses d'origines animale et végétale (tableau 8).

Des études postérieures à la situation de crise survenue en 1999 concernant la contamination de graisses incorporées dans des aliments pour volaille en Belgique montrent que les huiles végétales ne sont pas des matrices très contaminées en dioxines (Fraisse, 2003; Fernandez et al., 2004 ; Bordajandi et al., 2004 ; Baars et al., 2004). En effet, l'étude la plus complète conduit pour les huiles d'olive et de tournesol, groupe constitué d'une vingtaine d'échantillons, à des teneurs en moyenne de $0,25 \mathrm{pg} / \mathrm{g}$ (OMS-PCDD/F-TEQ), pour une limite européenne fixée à $0,75 \mathrm{pg} / \mathrm{g}$. Dans cette même étude, les teneurs en PCB-type dioxine sont en moyenne de l'ordre de $0,60 \mathrm{pg} / \mathrm{g}$ (OMS-PCB-TEQ), ce qui conduit à un total de $0,85 \mathrm{pg} / \mathrm{g}$ pour la somme dioxines et $P C B$ de type dioxine, pour une teneur maximale réglementaire de $1,5 \mathrm{pg} / \mathrm{g}$ (OMSPCDD/F-PCB-TEQ) (tableau 9).

Ce type de contaminants concerne principalement les huiles de poisson. Plusieurs études ont été réalisées sur l'élimination de ces polluants au cours du raffinage, notamment par la société DE SMET qui a développé plusieurs procédés assez poussés afin de respecter les limites fixées par la réglementation (tableau 8): ces procédés sont assez semblables à ceux mis en œuvre pour l'élimination des HAP et sont basés sur :

- un traitement sur CA éventuellement couplé à un prétraitement sur silice afin d'en améliorer l'efficacité ;

- un traitement de stripping (désodorisation autour de $190{ }^{\circ} \mathrm{C}$ avec injection de $2 \%$ de vapeur).

Avec ce type de traitement, DE SMET revendique la possibilité d'atteindre, à partir d'une huile brute de poisson contenant $5,3 \mathrm{pg} / \mathrm{g}$ de dioxines et $25,1 \mathrm{pg} / \mathrm{g}$ de PCB (soit un total de $30,4 \mathrm{pg} / \mathrm{g}$ ), une huile raffinée contenant $0,1 \mathrm{pg} / \mathrm{g}$ de dioxines et $1,6 \mathrm{pg} / \mathrm{g}$ de $P C B$ totaux, soit un total de $1,7 \mathrm{pg} / \mathrm{g}$ (limite réglementaire : $10 \mathrm{pg} / \mathrm{g}$ ).

Tableau 8. Extrait de l'annexe, section 5 (dioxines et $P C B^{a}$ ), du règlement $C E n^{\circ} 1881 / 2006$ concernant les corps gras d'origines animale et végétale.

\begin{tabular}{|c|c|c|c|}
\hline \multicolumn{2}{|c|}{ Denrées alimentaires } & \multicolumn{2}{|c|}{ Teneurs maximales ( $\mathrm{pg} / \mathrm{g}$ de graisses) } \\
\hline \multirow[t]{4}{*}{5.7} & Graisses des animaux suivants & $\begin{array}{l}\text { Somme des dioxines } \\
\text { (OMS-PCDD/F-TEQ) }^{\text {b }}\end{array}$ & $\begin{array}{l}\text { Somme des dioxines } \\
\text { et PCB de type dioxine } \\
\text { (OMS-PCDD/F-PCB-TEQ) }^{\text {b }}\end{array}$ \\
\hline & Bovins et ovins & 3,0 & 4,5 \\
\hline & Volailles & 2,0 & 4,0 \\
\hline & Porcs & 1,0 & 1,5 \\
\hline 5.8 & Graisses animales mélangées & 2,0 & 3,0 \\
\hline 5.9 & Huiles et graisses végétales & 0,75 & 1,5 \\
\hline 5.10 & $\begin{array}{l}\text { Huiles marines (huiles de corps et de foie de poisson, huiles d'autres } \\
\text { organismes marins destinées à la consommation humaine) }\end{array}$ & 2,0 & 10,0 \\
\hline
\end{tabular}

${ }^{a}$ Dioxines (somme des PCDD et PCDF, exprimée en équivalents toxiques de I'OMS, après application des TEF-OMS [facteurs d'équivalence toxique]) et somme des dioxines et $\mathrm{PCB}$ de type dioxine (somme des PCDD, PCDF et PCB, exprimée en équivalents toxiques de l'OMS, après application des TEF-OMS). Les TEF-OMS pour une évaluation des risques encourus par l'homme fondée sur les conclusions de la réunion de l'OMS tenue à Stockholm (Suède), du 15 au 18 juin 1997 (Van den Berg et al. Facteurs d'équivalence toxique (TEF) pour les PCB, PCDD et PCDF, applicables à I'homme, la faune et la flore. Environ Health Perspect 1998 : 106(12); 775). Valeurs TEFOMS données en fin d'annexe du règlement (CE) $n^{\circ} 1881 / 2006$.

${ }^{b}$ Concentrations supérieures : on calcule les concentrations supérieures en supposant que toutes les valeurs des différents congénères au-dessous de la limite de quantification sont égales à la limite de quantification. 
Tableau 9. Niveaux de contamination en dioxines et PCB de type dioxine. Données bibliographiques.

\begin{tabular}{|c|c|c|c|c|}
\hline \multirow[t]{2}{*}{ Références bibliographiques } & \multirow{2}{*}{$\begin{array}{l}\text { Nature et (nombre) } \\
\text { des échantillons }\end{array}$} & \multicolumn{3}{|c|}{ Teneurs (pg/g) [OMS-TEQ ou WHO-TEQ] } \\
\hline & & $\begin{array}{l}\text { Dioxines } \\
\text { (OMS-PCDD/F-TEQ) }\end{array}$ & $\begin{array}{l}\text { PCB de type dioxine } \\
\text { (OMS- PCB-TEQ) }\end{array}$ & $\begin{array}{l}\text { Dioxines + PCB de type dioxine } \\
\text { (OMS-PCDD/F-PCB-TEQ) }\end{array}$ \\
\hline \multirow[t]{4}{*}{ Fraisse, 2003} & Huile de tournesol (1) & 0,05 & 0,004 & 0,054 \\
\hline & Huile de colza (1) & 0,02 & 0,000 & 0,020 \\
\hline & Huile d'arachide (1) & 0,04 & 0,004 & 0,044 \\
\hline & Huile de palme (1) & 0,03 & 0,004 & 0,034 \\
\hline Fernandez et al., 2004 & Huiles d'olive et de tournesol (20) & 0,25 & 0,60 & 0,85 \\
\hline \multirow[t]{2}{*}{ Bordajandi et al., 2004} & Huile de tournesol (?) & 0,34 & 1,70 & 2,04 \\
\hline & Huile d'olive (?) & 0,29 & 0,42 & 0,71 \\
\hline Baars et al., 2004 & Huile végétale (?) & & & 0,18 \\
\hline
\end{tabular}

Tableau 10. Caractéristiques physicochimiques des phtalates utilisés pour le dopage des huiles.

\begin{tabular}{|c|c|c|c|}
\hline & DIDP di-isodécyl phtalate & DEHP di-éthylhexyl phtalate & BBP butyl-benzyl phtalate \\
\hline Formule chimique & $\mathrm{C}_{28} \mathrm{H}_{46} \mathrm{O}_{4}$ & $\mathrm{C}_{24} \mathrm{H}_{38} \mathrm{O}_{4}$ & $\mathrm{C}_{19} \mathrm{H}_{20} \mathrm{O}_{4}$ \\
\hline $\mathrm{N}^{\circ}{ }^{\circ}$ es cas & $26761-40-0$ & $117-81-7$ & $85-68-7$ \\
\hline Masse molaire $(\mathrm{g} / \mathrm{mol})$ & 446,7 & 390,6 & 312,36 \\
\hline Aspect & $\begin{array}{l}\text { Liquide visqueux, limpide, } \\
\text { sans couleur et odeur faible }\end{array}$ & Liquide visqueux incolore & Liquide incolore, huileux \\
\hline Densité à $20^{\circ} \mathrm{C}$ & $0,965^{* *}$ & $0,981^{* *} / 0,983^{* * *} / 0,986^{*}$ & $1,1^{* *} / 1,1^{*}$ \\
\hline Solubilité dans l'eau & Nulle* & Nulle* & Nulle*/** \\
\hline Tension de vapeur à $200^{\circ} \mathrm{C}$ & $147 \mathrm{~Pa}^{*}$ & $160 \mathrm{~Pa}^{\star * *}$ & Pas de données \\
\hline Tension de vapeur à $20^{\circ} \mathrm{C}$ & $2,8 \times 10^{-5} \mathrm{~Pa}^{* * *}$ (estimation) & $0,001 \mathrm{kPa}^{*}\left(\right.$ ou $\left.10^{-6} \mathrm{~Pa}\right)$ & $<0,1 \mathrm{~Pa}^{*}$ \\
\hline Tension de vapeur à $25^{\circ} \mathrm{C}$ & $5,1 \times 10^{-5} \mathrm{~Pa}^{\star \star *}$ (estimation) & env. $10^{-6} \mathrm{~Pa}^{* * *}$ & Pas de données \\
\hline \multirow[t]{2}{*}{ Point d'éclair } & $229^{\circ} \mathrm{C}^{*}$ & $200^{\circ} \mathrm{C}^{* * *}$ & $>110^{\circ} \mathrm{C}^{* *}$ \\
\hline & $275^{\circ} \mathrm{C}^{* *}$ & $207^{\circ} C^{* *}$ & $199^{\circ} \mathrm{C}^{*}$ \\
\hline Coefficient de partage octanol/eau & $\log P_{O / E}: 4,9^{*}$ & $\log P_{O / E}: 5,03^{*}$ & $\log P_{O / E}: 4,77^{*}$ \\
\hline Commentaires & $\begin{array}{l}\text { *Données issues de la fiche } \\
\text { internationale de sécurité } \\
\text { chimique } 1999 \\
{ }^{* \star} \text { Données issues de la fiche de données } \\
\text { de sécurité CLUZEAU }(15 / 02 / 2006) \\
\text { *** Données issues de la fiche } \\
\text { toxicologique }{ }^{\circ} 246 \text { INRS }\end{array}$ & $\begin{array}{l}\text { * Données issues de la fiche } \\
\text { internationale de sécurité } \\
\text { chimique } 2001 \\
\text { ** Données issues de la fiche de données } \\
\text { de sécurité CLUZEAU }(03 / 02 / 2006) \\
\text { *** Données issues de la fiche } \\
\text { toxicologique }{ }^{\circ} 161 \text { INRS }\end{array}$ & $\begin{array}{l}\text { * Données issues de la fiche } \\
\text { internationale de sécurité } \\
\text { chimique } 1998 \\
\text { ** Données issues de la fiche de } \\
\text { données de sécurité CLUZEAU } \\
(17 / 01 / 2007)\end{array}$ \\
\hline
\end{tabular}

\section{Élimination de « nouveaux » contaminants}

\section{Phtalates}

La nature, l'origine et le statut réglementaire de ce type de contaminant sont rappelés dans ce dossier par l'article de Lacoste et al. ${ }^{15}$

\footnotetext{
15 « Détection de contaminants dans les huiles végétales : bilan à fin 2009 ».
}

Depuis 2007, I'ITERG conduit des travaux sur l'élimination des phtalates au cours du raffinage. Le tableau 10 résume les caractéristiques physicochimiques des phtalates retenus pour ces études. Les premiers essais ont été réalisés sur de petites quantités (environ $5 \mathrm{~kg}$ ) d'huile de tournesol oléique ${ }^{16}$ dopée à 5 et $50 \mathrm{mg} / \mathrm{kg}$ en butyl-benzyl phtalate (BBP), di-éthylhexyl

\footnotetext{
${ }^{16}$ Fournie par la Société CREOL (huile brute obtenue par cuisson et pression).
}

phtalate (DEHP) et di-isodécyl-phtalate (DIDP) soumises aux différentes étapes d'un raffinage chimique et d'une distillation neutralisante (raffinage "physique ») en faisant varier les paramètres de la désodorisation ou de la distillation (température, temps de séjour, quantité de vapeur injectée); les quantités de phtalates résiduels ont été contrôlés aux différentes étapes de chaque raffinage ${ }^{17}$.

\footnotetext{
${ }^{17}$ Analyses réalisées par l'unité analyse de l'ITERG.
} 
Les premières observations montrent clairement que les premières étapes du raffinage (physique et chimique) ne réduisent pas la teneur en phtalates des huiles dopées, quel que soit le niveau du dopage, et que leur élimination se fait essentiellement à l'étape de désodorisation (raffinage chimique) ou à la désacidification (raffinage physique).

En raffinage chimique, il apparaît que la température de la désodorisation et la quantité de vapeur injectée sont des paramètres importants qui conditionnent l'élimination des phtalates (figures $6 A$ et $B$ ).

En raffinage physique, il semble que, une fois la température optimale déterminée, son augmentation n'accroisse pas significativement le taux d'élimination (figure 7).

Le BBP apparaît plus aisément éliminable que le DEHP, même si pour ce dernier, des conditions appropriées conduisent à des taux d'élimination de 80 à $90 \%$; cette nuance dans le comportement de ces deux composés peut s'expliquer par leurs caractéristiques physicochimiques rappelées dans le tableau 10: masse molaire, tensions de vapeur et coefficient de partage octanol/eau ${ }^{18}$. Le DIDP est le plus difficile à éliminer, mais l'influence des conditions de désodorisation sur son élimination est difficile à caractériser, étant donné les difficultés analytiques rencontrées sur ce composé $^{19}$ dont la limite de quantification reste très élevée en comparaison de celle des BBP et DEHP ( $<1 \mathrm{mg} / \mathrm{kg})$.

L'étude a été a poursuivie par des essais semi-industriels menés en atelier pilote de raffinage (capacité de 1 tonne par batch). Un essai de raffinage chimique et un essai de raffinage "physique» ont été réalisés sur $400 \mathrm{~kg}$ d'huile de tournesol oléique ${ }^{16}$ dopée à $50 \mathrm{mg} / \mathrm{kg}$ en BBP et DEHP et à $10 \mathrm{mg} / \mathrm{kg}$ en DIDP.

\section{Conditions du raffinage physique}

- Prétraitement : 0,1\% d'acide phosphorique $75 \%, 70{ }^{\circ} \mathrm{C}, 45$ minutes, puis traitement au Tonsil 210 FF (1\%), 30 minutes sous vide et filtration sur Büchner ;

- désacidification : injection de vapeur d'eau, sous vide inférieur à $5 \mathrm{mb}$, deux et quatre heures à $240{ }^{\circ} \mathrm{C}$.

\footnotetext{
${ }^{18}$ Traduisant le caractère plus ou moins lipophile d'un composé et, en conséquence, sa plus ou moins grande facilité d'élimination.

${ }^{19}$ Qui est en réalité un ensemble de composés variant par la longueur de leur chaîne latérale et conduisant à une mauvaise résolution chromatographique (voir article Lacoste et al., dans ce dossier).
}

Conditions du raffinage chimique phosphorique $75 \%, 70{ }^{\circ} \mathrm{C}, 45$ minutes;

- neutralisation à la soude avec un excès de $10 \%$ : 30 minutes, à $70^{\circ} \mathrm{C}$;

- lavages (jusqu'à pH = 7) ;

- séchage sous vide : une heure à $85^{\circ} \mathrm{C}$;
- Conditionnement acide : 0,1\% d'acide
- décoloration : $1 \%$ de Tonsil optimum 210 $\mathrm{FF}, 85^{\circ} \mathrm{C}$, sous vide pendant 40 minutes et filtration sur Büchner ;

- désodorisation : injection de vapeur d'eau, sous vide inférieur à $5 \mathrm{mb}$, deux et quatre heures à $200^{\circ} \mathrm{C}$.

\section{A}

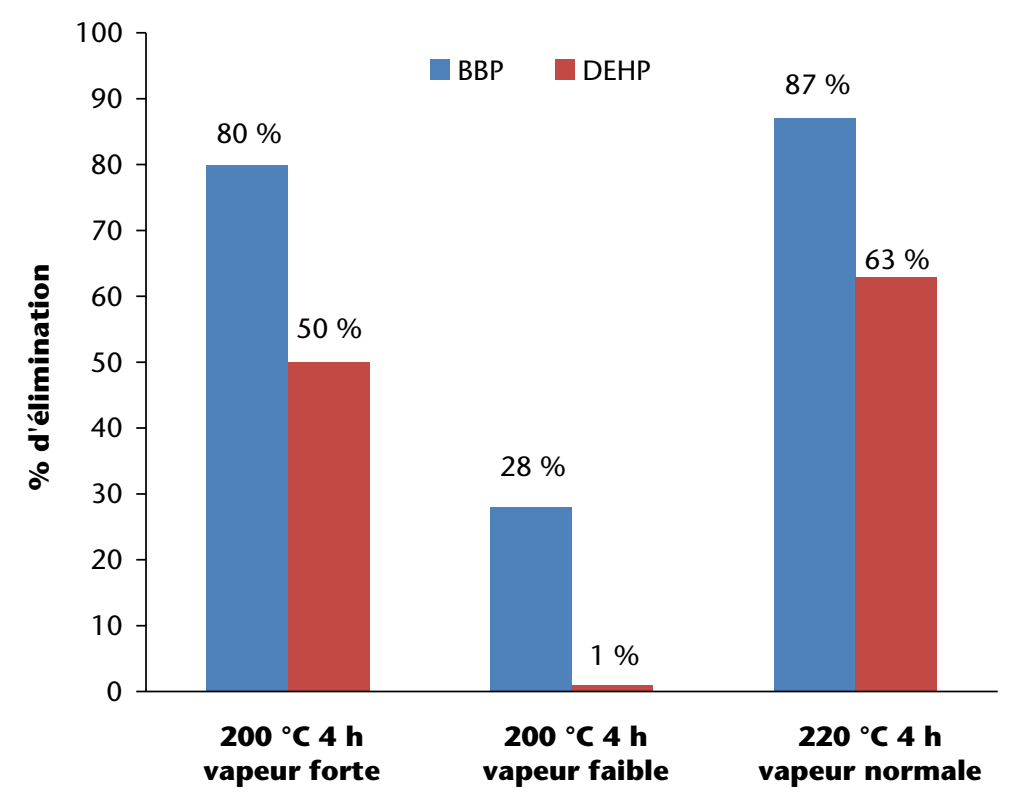

B

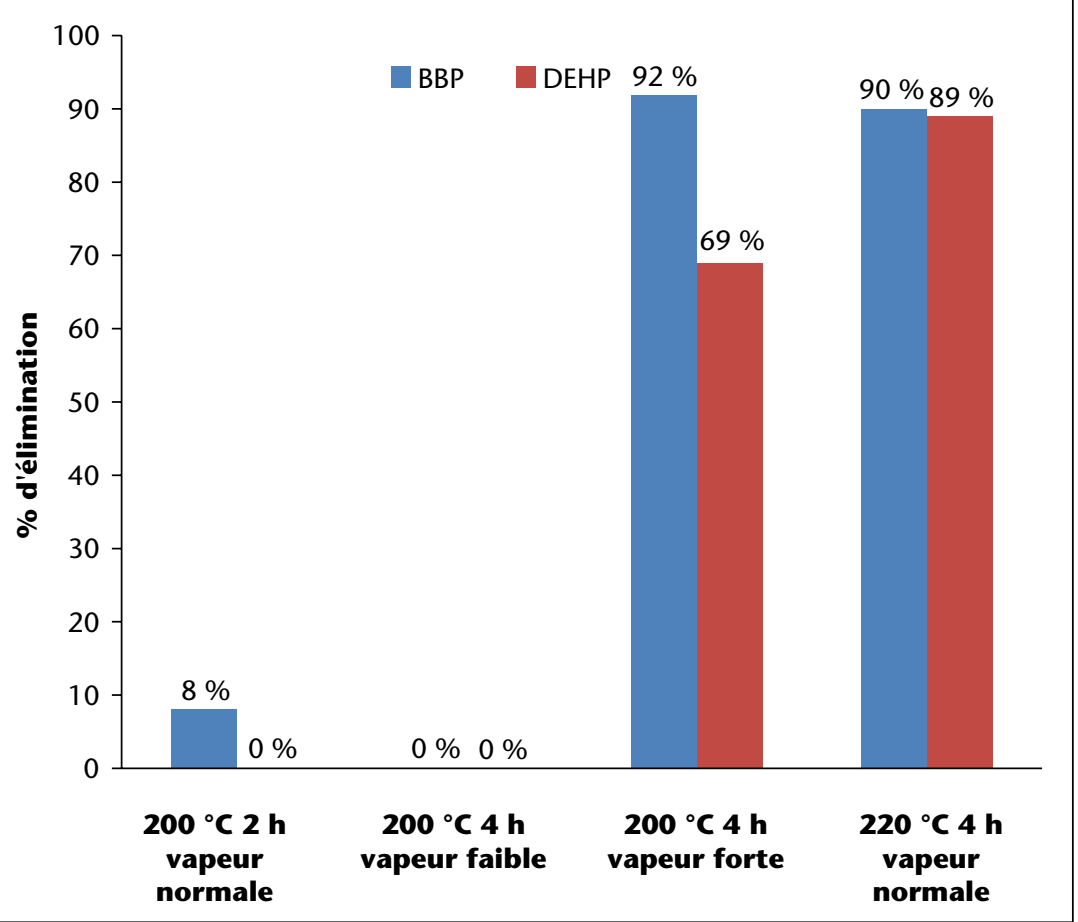

Figure 6. Pourcentage d'élimination des phtalates (BBP et DEHP) présents dans une huile de tournesol oléique dopée à $50 \mathrm{mg} / \mathrm{kg}$ (A) et à $5 \mathrm{mg} / \mathrm{kg}$ (B) au cours de la désodorisation d'un raffinage chimique réalisé sur de petites quantités (environ $5 \mathrm{~kg}$ ). Influence des paramètres température et quantité de vapeur injectée. 


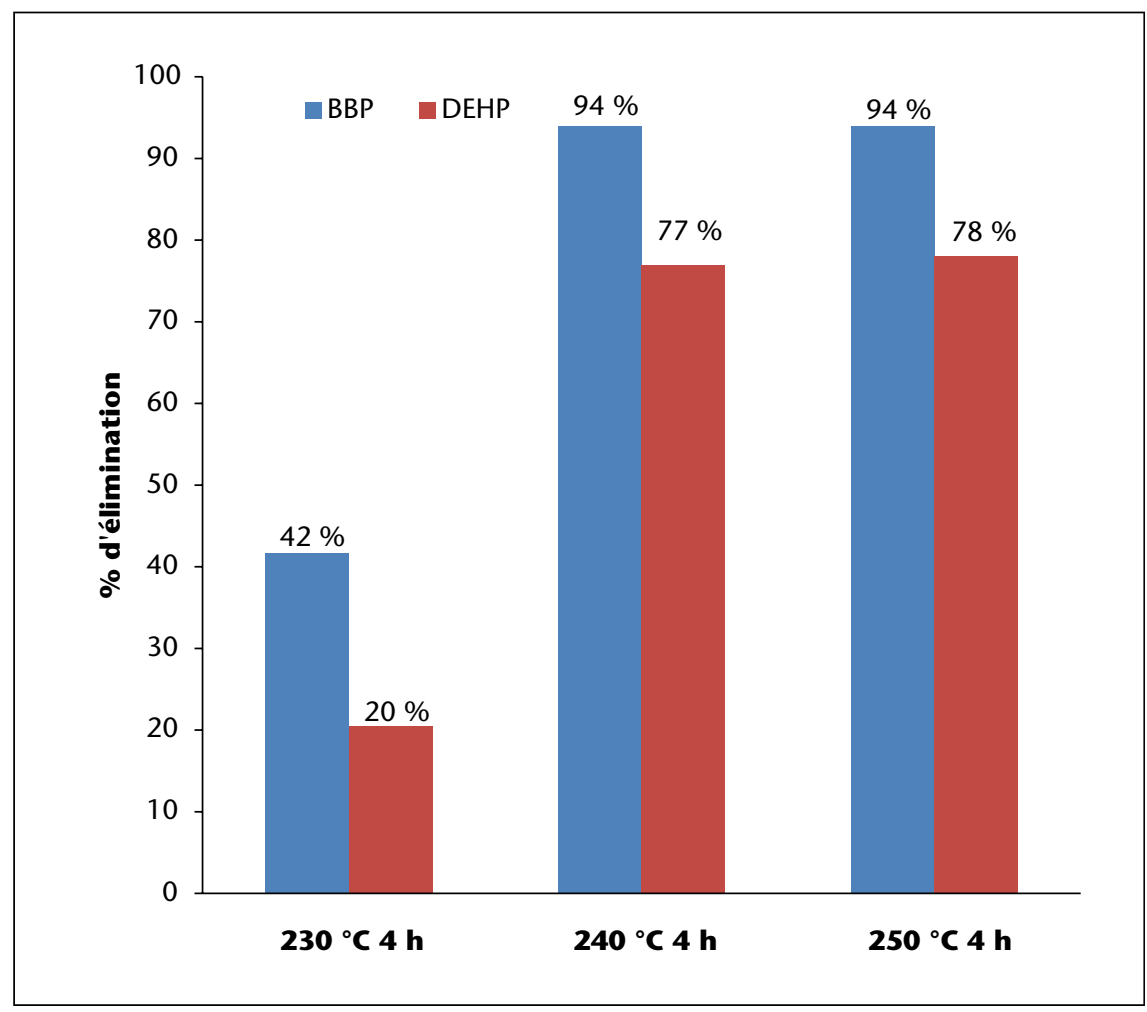

Figure 7. Pourcentage d'élimination des phtalates (BBP et DEHP) présents dans une huile de tournesol oléique dopée à $50 \mathrm{mg} / \mathrm{kg}$ au cours de la désacidification d'un raffinage "physique " réalisé sur de petites quantités (environ $5 \mathrm{~kg}$ ). Influence du paramètre température.

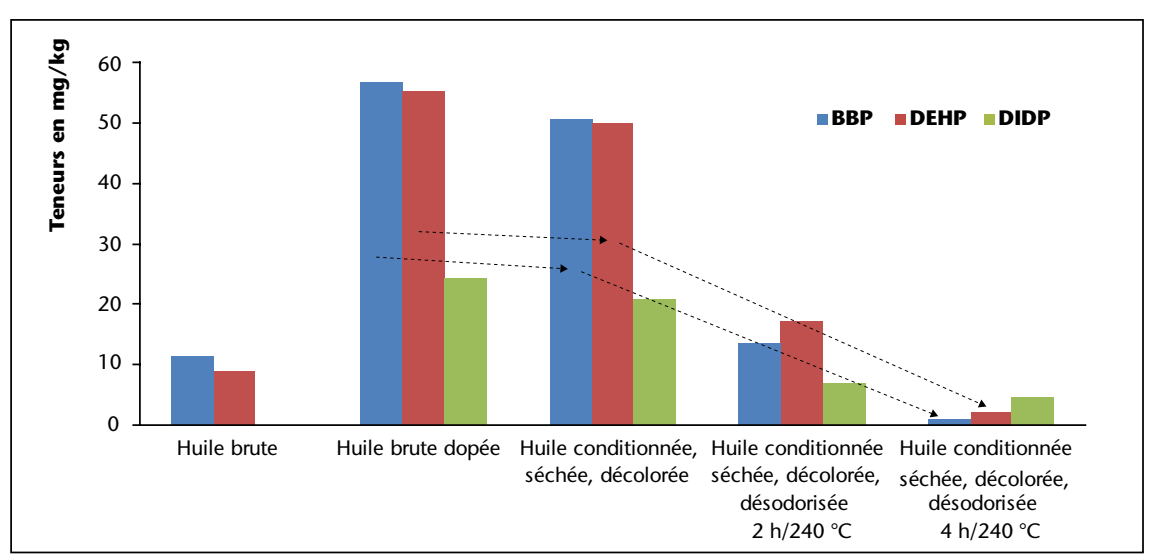

Figure 8. Évolution de la teneur en phtalates d'une huile dopée au cours du raffinage physique réalisé en atelier de raffinage (400 kg). Niveaux de dopage: BBP, DEHP, $50 \mathrm{mg} / \mathrm{kg} ;$ DIDP, $10 \mathrm{mg} / \mathrm{kg}$.

Des échantillons ont été prélevés à chaque étape pour détermination de la teneur en phtalates ${ }^{17}$.

Les résultats de ces essais semi-industriels confirment les premiers essais: premières étapes de raffinage sans effet sur l'élimination des phtalates qui n'est possible qu'à l'étape de désodorisation (raffinage chimique, faiblement) ou à l'étape de désacidification (beau-
En deux heures, toujours à $240{ }^{\circ} \mathrm{C}, 76 \% \mathrm{du}$ BBP (TR $\approx 14 \mathrm{mg} / \mathrm{kg})$ et $68 \%$ du DEHP ( $T R \approx 17 \mathrm{mg} / \mathrm{kg}$ ) sont éliminés.

Les résultats obtenus lors des premiers essais sont donc confirmés et sont même encore meilleurs à l'échelle semi-industrielle pour quatre heures de désodorisation à $240^{\circ} \mathrm{C}$.

Comme pour les premiers essais, le dosage du DIDP n'étant pas optimisé, il est impossible de conclure sur les résultats obtenus.

Raffinage chimique, huile dopée à $50 \mathrm{mg} / \mathrm{kg}$ (BBP et DEHP) et $10 \mathrm{mg} / \mathrm{kg}$ (DIDP)

Les résultats sont présentés sur la figure 9 : une désodorisation de quatre heures à $200^{\circ} \mathrm{C}$ avec une injection de vapeur moyenne ( $1 \% /$ heure) permet de diminuer la teneur en BBP de $45 \%$ $(T R \approx 28 \mathrm{mg} / \mathrm{kg}$ ) et la teneur en DEHP de seulement $17 \%$ (TR $\approx 41 \mathrm{mg} / \mathrm{kg}$ ). Deux heures de désodorisation menée dans les mêmes conditions (température, vapeur) conduisent à une diminution encore plus faible des teneurs en BBP et DEHP.

L'importance du paramètre "quantité de vapeur injectée » sur la diminution des teneurs en BBP et DEHP en raffinage chimique (voir premiers essais, plus haut) paraît se confirmer au terme de ces nouveaux essais. Ce point sera confirmé par de nouveaux essais qui permettront également d'aborder le cas d'autres molécules de phtalates, comme par exemple le DBP (di-butyl phtalate), suite à la révision de la liste des produits à substituer établie en lien avec les procédures REACH par l'Agence européenne des produits chimiques $\left(E \mathrm{CHA}^{20}\right)$ en 2009.

\section{Esters de 3-monochloropropane-1, 2-diol (3-MCPD)}

La nature, le contexte relatif à ce nouveau contaminant potentiel dont la présence a été récemment signalée dans des huiles végétales raffinées ainsi que les difficultés analytiques rencontrées pour sa détermination (se traduisant par des approches méthodologiques fort différentes) sont rappelés dans ce dossier par I'article de Lacoste et al., Détection de contaminants dans les huiles végétales : bilan à fin 2009. Les données concernant l'évolution de la teneur de ce polluant (tel quel ou sous forme d'esters d'acides gras) au cours du raffinage des huiles sont dans l'état actuel des connaissances incertaines. Certains travaux ne sont pas probants et concernent soit des huiles de friture, soit des huiles extraites de denrées alimentaires.

\section{Raffinage physique, huile dopée à $50 \mathrm{mg} / \mathrm{kg}$} (BBP et DEHP) et $10 \mathrm{mg} / \mathrm{kg}$ (DIDP)

Les résultats sont présentés sur la figure $8: 98 \%$ du BBP (teneur résiduelle $[T R]<1 \mathrm{mg} / \mathrm{kg}$ ) et $96 \%$ du DEHP (TR $\approx 2 \mathrm{mg} / \mathrm{kg}$ ) sont éliminés en quatre heures de désodorisation à $240^{\circ} \mathrm{C}$.

\footnotetext{
${ }^{20} \mathrm{http}: / /$ ec.europa.eu/enterprise/sectors/chemicals/ files/markrestr/echa_plan_phthalates_091026_en.pdf http://echa.europa.eu/home_fr.asp et http://www. synamap.fr/epi-et-phtalates.htm
} 


\section{Teneurs en $\mathbf{~ m g} / \mathbf{k g}$}

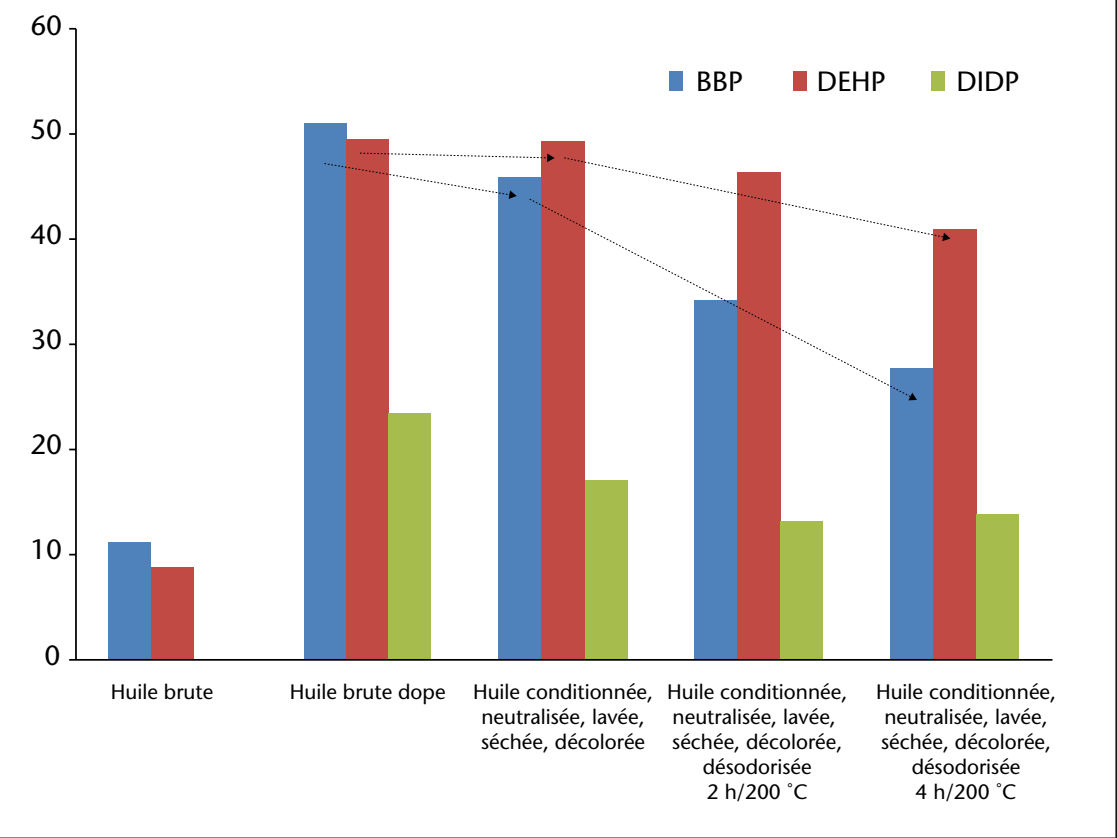

Figure 9. Évolution de la teneur en phtalates d'une huile dopée au cours du raffinage chimique réalisé en atelier de raffinage (400 kg). Niveaux de dopage : BBP, DEHP, $50 \mathrm{mg} / \mathrm{kg} ;$ DIDP, $10 \mathrm{mg} / \mathrm{kg}$.
Des résultats intéressants car réalisés sur des échantillons industriels simplement prélevés à diverses étapes du raffinage sont reportés par Zelinková et al. (2006) sur de l'huile de colza, qui citent une diminution dès le dégommage : de 20 à 11 et de 990 à moins de $300 \mu \mathrm{g} / \mathrm{kg}$ respectivement pour le 3-MCPD libre et lié ; les étapes de décoloration et désodorisation permettant $d$ 'atteindre respectivement moins de $300 \mu \mathrm{g} / \mathrm{kg}$ et moins de $9 \mu \mathrm{g} / \mathrm{kg}$.

Plus récemment, Franke et al. (2009), lors $d^{\prime}$ essais de raffinage chimique à l'échelle du laboratoire sur des huiles de palme et de colza, identifient que la désodorisation est la seule étape conduisant à une augmentation des teneurs en esters de 3-MCPD, déterminées par la méthode de Weisshaar (2008); les mêmes auteurs relient l'origine de la formation des esters de 3-MCPD à la présence de diglycérides et à la quantité de chlore total mesurée dans les huiles. Ces conclusions sont remises en cause par Collison (2010) ${ }^{21}$ qui montre les biais de la méthode de Weisshaar et préconise d'orienter les futurs travaux de recherche analytique vers des méthodes d'analyse directe (couplage CLHP/SM) des esters de 3-MCPD ou des monoesters de glycidol, l'occurrence

Tableau 11. Raffinage et élimination des contaminants. Résumé des étapes efficaces par type de contaminant.

\begin{tabular}{|c|c|c|}
\hline Composés à éliminer & $\begin{array}{l}\text { Étape(s) efficace(s) } \\
\text { du raffinage }\end{array}$ & Commentaires \\
\hline Traces de solvants & Décoloration, désodorisation & Effets du vide \\
\hline Traces métalliques & $\begin{array}{l}\text { Chimique : neutralisation et décoloration } \\
\text { Physique : prétraitement/adsorbant }\end{array}$ & Adsorption \\
\hline Pesticides (PEST) & $\begin{array}{l}\text { Conditionnement acide } \\
\text { Neutralisation } \\
\text { Lavages } \\
\text { Décoloration } \\
\text { Désodorisation** }\end{array}$ & $\begin{array}{l}\text { Pour PEST instables en milieu acide (pyrimiphos méthyl) } \\
\text { Pour PEST instables en milieu basique et solubles dans l'eau } \\
\text { (dichlorvos, malathion) } \\
\text { Pour PEST solubles dans l'eau [f(solubilité)] } \\
\text { PEST à site cationique } \\
\text { L'étape décisive le plus souvent }\end{array}$ \\
\hline HAP & $\begin{array}{l}\text { Décoloration** } \\
\text { Désodorisation* }\end{array}$ & $\begin{array}{l}\text { Surtout HAP lourds; importance du choix du charbon actif, } \\
\text { mode d'activation, quantité et température de décoloration } \\
\text { Pour HAP légers seulement }\end{array}$ \\
\hline Dioxines/PCB & $\begin{array}{l}\text { Traitement spécifique DE SMET**: } \\
\text { Charbon actif } \\
\text { Stripping à la désodorisation }\end{array}$ & $\begin{array}{l}\text { Concerne surtout les huiles de poisson } \\
\text { + au besoin prétraitement silice } \\
190^{\circ} \mathrm{C}, 2 \% \text { de vapeur }\end{array}$ \\
\hline Phtalates & Désodorisation/désacidification & Études ITERG en cours \\
\hline 3-MCPD & Enjeu : formation au cours du raffinage? & $\begin{array}{l}\text { Priorité : résoudre les questions liées aux méthodes d'analyse } \\
\text { - Études en cours }\end{array}$ \\
\hline Huiles minérales légères & Désodorisation & Par stripping vapeur ; efficacité non systématique \\
\hline Huiles minérales lourdes & Pas d'étape efficace & \\
\hline \multirow[t]{2}{*}{ Mycotoxines (aflatoxines) } & Neutralisation** & Action de la soude \\
\hline & Décoloration* & Parfait l'élimination \\
\hline
\end{tabular}


de ces derniers dans des huiles végétales raffinées lui paraissant être plus significative (et corrélée à la présence de diglycérides) que celle des premiers. Par ailleurs, Collison montre que des esters de 3-MCPD peuvent se former au contact d'adsorbants activés à l'acide chlorhydrique.

L'unité de technologie de I'ITERG a inscrit l'étude des esters de 3-MCPD en cours de raffinage au programme de ses travaux 2010, en lien avec la recherche analytique menée par l'unité analyse.

\section{Conclusion}

Ce large tour d'horizon montre globalement que le procédé de raffinage (par voie chimique ou "physique») est le plus souvent très efficace pour garantir la sécurité sanitaire des huiles et corps gras raffinés et leur conformité avec les exigences réglementaires. Le tableau 11 résume pour chaque contaminant les étapes les plus efficaces du raffinage.

Les conditions d'élimination optimales de certaines catégories de contaminants sont maintenant bien connues (solvants résiduels, traces métalliques, HAP, la majorité des substances phytosanitaires susceptibles de contaminer les oléagineux, etc.); mais d'autres, notamment les nouveaux contaminants potentiels plus ou moins résistants au raffinage, réclament des études spécifiques et une optimisation réaliste des procédés: nouvelles substances actives phytosanitaires (ce fut le cas de la deltaméthrine, plutôt résistante à l'élimination), évolution de la réglementation des contaminants connus (cas des HAP en 2010, avec de nouvelles molécules à surveiller en plus du B(a)P), cas des phtalates lourds et plus récemment encore la question des esters de 3-MCPD ; dans ces deux derniers cas, la première étape à franchir est la recherche de méthodes d'analyse donnant des résultats fiables dont l'absence conduit à des interprétations erronées.

C'est pourquoi, I'identification de critères physicochimiques indicatifs d'un probable comportement de composés potentiellement contaminants au cours des procédés de raffinage constitue un outil d'orientation préalable intéressant $^{22}$.

Par ailleurs, les travaux à venir devront étudier les produits de transformation (notamment des pesticides) pouvant se former lors des opérations de raffinage ainsi que le contrôle de leur élimination par ces mêmes opérations. Enfin, il faut rappeler que la qualité des huiles et corps gras raffinés intègre le maintien ou l'amélioration des caractéristiques organoleptiques, nutritionnelles et la stabilité en conservation, d'une part, et, d'autre part, l'élimination des contaminants tout en évitant ou maîtrisant la formation de nouveaux composés indésirables. Pour chaque composé qu'il s'agit d'éliminer, les enjeux technologiques du raffinage résident au carrefour de ces exigences.

\section{RÉFÉRENCES}

Couteux A, Salaün C. ACTA Index phytosanitaire. $45^{\mathrm{e}}$ édition, 2009.

Baars Al, Bakker MI, Baumann RA, et al. Dioxins, dioxin-like PCBs and non-dioxin-like PCBs in foodstuffs: occurrence and dietary intake in The Netherlands. Toxicol Lett $2004 ; 151$ : 51-61.

Bordajandi LR, Gomez G, Abad E, et al. Survey of persistent organochlorine contaminants, heavy metals in food samples from Huelva (Spain): levels and health implications. J Agric Food Chem 2004; 52 : 992-1001.

Castera A. Traces métalliques. In : Manuel des corps gras. Tome 2, chap. XIV, p. 1243-55. Coordonnateur A. Karleskind. Paris : Ed. Lavoisier Tec\&Doc, 1992

Collison MW. Direct determination of MCPB esters and glycidyl esters by LCMS. Communication OVID Meeting, 25/01/2010, Berlin.

Dauguet $S$, Lacoste $F$, Ticot $B$, et al. La filière oléagineuse se mobilise autour de la problématique des résidus d'insecticides. OCL $2006 ; 13$ : 373-7.

Denise J. Le raffinage des corps gras. WesthoekEditions, Les Editions du Beffroi, 1983; (ouvrage épuisé).

DGCCRF. Note de service $n^{\circ}$ du 12 décembre 1990, basée sur un avis du Conseil supérieur d'hygiène publique de France, 1990.
Dugo G, La Pera L, La Torre GL, Giuffrida D. Determination of $\mathrm{Cd}(\mathrm{II}), \mathrm{Cu}(\mathrm{II}), \mathrm{Pb}(\mathrm{II})$, and $\mathrm{Zn}(\mathrm{II})$ content in commercial vegetable oils using derivative potentiometric stripping analysis. Food Chem 2004; 87 : 639-45.

Fernandez MA, Gomara B, Bordajandi LR, et al. Dietary intakes of polychlorinated dibenzo-p-dioxins, dibenzofurans and dioxin-like polychlorinated biphenyls in Spain. Food Addit Contam 2004; 21 : 983-91.

Fraisse $D$. Les dioxines et les $P C B$ type-dioxine dans les aliments gras. OCL $2003 ; 10: 280-6$.

Franke K, Strijowski U, Fleck G, Pudel F. Influence of chemical refining process and oil type on bound 3-chloro-1,2-propanediol contents in palm oil and rapeseed oil. Food Sci Technol 2009 ; 42 : 1751-4.

Jouve JL. La qualité microbiologique des aliments. Mâ̂trise et critères. $2^{\mathrm{e}}$ partie, chap. 7, p. 124-25 Ed. CNRS-CNERNA / Polytechnica, 1993.

Lacoste F, Lechat $H$, Pages $X$, et al. Contrôle des composés indésirables dans les huiles végétales et mise en place d'observatoires. OCL 2005 ; 12 : 372-7.

Pages X, Laur J, Birot C, Arnaud JN, Lacoste F. Influence of the refining process on pesticide residues removal. Communication au congrès Euro Fed Lipid 2004; Edinburgh (UK)

Pages X, Birot C, Escabasse J, Fazeuilh S, Hermon M, Lacoste F. Refining: removal of pesticide residues. Communication au congrès Euro Fed Lipid 2006; Madrid (S).

Ruiz Mendez MV, de la Rosa IP, Jiménez Márquez A, et al. Elimination of pesticides in olive oil by refining using bleaching and deodorization. Food Addit Contam $2005 ; 22: 23-30$.

The Pesticide Manual., Ed. C.D.S Tomlin, $14^{\text {th }}$ edition, British Crop Production Council (BPC) 2006.

Van Duijn G. Industrial experiences with pesticide removal during edible oil refining. Eur J Lipid Sci Technol $2008 ; 110$ : 982-9.

Weisshaar R. Determination of total 3-chloropropane-1,2-diol (3-MCPD) in edible oils by cleavage of MCPD esters with sodium methoxide. Eur / Lipid SCi Technol 2008 ; 110 : 183-6.

Zelinková Z, Svejkovská B, Velisek J, Dolezal M. Fatty acid esters of 3-chloropropane-1,2-diol in edible oils. Food Addit Contam $2006 ; 23: 1290-8$.

\footnotetext{
${ }^{21}$ Voir article Lacoste et al., dans ce dossier.

22 Hydrosolubilité ou caractère lipophile (solubilité dans l'eau et $\log P\left(K_{\mathrm{O} / \mathrm{E}}\right)$, poids moléculaire et tension de vapeur.
} 